\title{
A Theoretical Study of Phosphodiester Hydrolysis and Transesterification Catalyzed By an Unsymmetric Biomimetic Dizinc Complex
}

\author{
Henrik Daver ${ }^{a}$, Biswanath Das ${ }^{b}$, Ebbe Nordlander ${ }^{b}$ Fahmi Himo $^{a, *}$
}

a. Department of Organic Chemistry, Arrhenius Laboratory, Stockholm University, SE-106 91 Stockholm, Sweden

b. Inorganic Chemistry Research Group, Chemical Physics, Center for Chemistry and Chemical Engineering, Lund University, Box 124, SE-221 00 Lund, Sweden

* Corresponding author. Telephone number: +46 8 161094; E-mail address: fahmi.himo@su.se

\section{Contents}

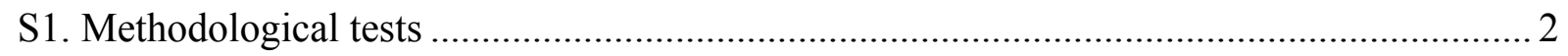

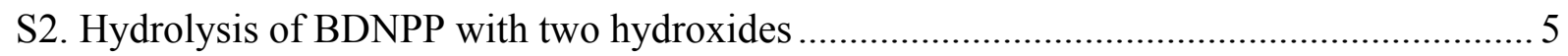

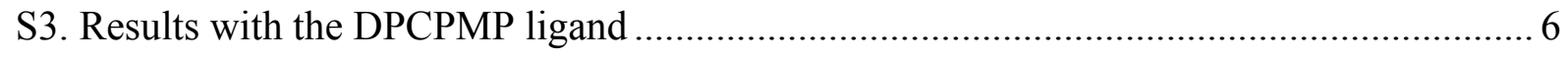

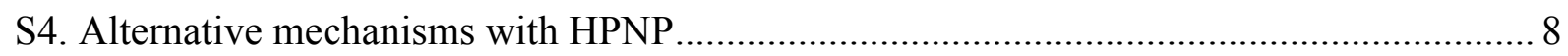

S5. Calculated absolute energies and energy corrections ................................................. 11

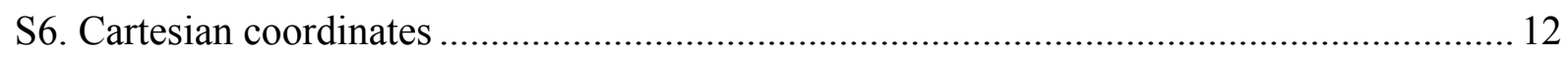




\section{S1. Methodological tests}

The sensitivity of the results to the choice of DFT method and solvation model has been tested.

\section{S1.A Results with different DFT methods}

Single-point energy calculations using different functionals were performed on the optimized geometries with the same basis set composition as the geometry optimizations. The corrections for large basis set, solvation, and free energy were taken from the B3LYP calculations. For the case in which the effect of the low-lying frequencies on the free energies was assessed, the free energy correction was calculated using standard rigid-rotor harmonic oscillator approximation (RRHO), i.e. without special treatment of vibrational modes with low wavenumbers .

The results are given in Table S1 for BDNPP and Table S2 for HPNP.

Table S1. Calculated free energies $(\mathrm{kcal} / \mathrm{mol})$ relative to $\boldsymbol{C} \cdot \mathbf{1}$ for hydrolysis of BDNPP.

\begin{tabular}{|c|c|c|c|c|c|c|c|c|}
\hline Method & $C \cdot 1 B$ & 1-TS1 & $C \cdot 1$ Int & 1-TS2 & $C \cdot 2 \cdot 3$ & $\mathrm{C} \cdot 2^{+}$ & $\begin{array}{l}\text { Reaction } \\
\text { (1) }\end{array}$ & $\Delta G^{\ddagger 1}$ \\
\hline B3LYP-D3 & 7.2 & 10.8 & 9.4 & 8.6 & -20.2 & -18.4 & -10.0 & 21.0 \\
\hline B3LYP-D3 (RRHO) ${ }^{\mathrm{a}}$ & 8.1 & 11.7 & 10.2 & 8.7 & -19.0 & -16.2 & -7.6 & 23.1 \\
\hline B3LYP ${ }^{b}$ & 9.3 & 11.5 & 10.1 & 6.9 & -18.3 & -30.6 & -14.1 & 28.0 \\
\hline B3LYP*-D3 ${ }^{c}$ & 6.9 & 10.1 & 8.6 & 7.9 & -20.8 & -17.4 & -10.6 & 20.3 \\
\hline $\mathrm{M}^{\mathrm{d}} \mathrm{d}^{\mathrm{d}}$ & 7.8 & 9.4 & 8.2 & 5.9 & -22.4 & -25.6 & -10.0 & 25.0 \\
\hline M06-D3 & 7.8 & 10.4 & 9.3 & 7.5 & -22.0 & -20.9 & -8.7 & 23.7 \\
\hline M06- $\mathrm{L}^{\mathrm{e}}$ & 10.4 & 9.7 & 8.2 & 6.9 & -21.9 & -24.7 & -8.7 & 26.4 \\
\hline
\end{tabular}

a. Entropies calculated using standard rigid-rotor harmonic oscillator approximation, i.e. without special treatment of vibrational modes with low wavenumbers.

b. Calculated with standard B3LYP functional, i.e.not including the DFT-D3 dispersion correction.

c. $15 \%$ Hartree-Fock exchange is used, as has been suggested for complexes containing transition metals, see Reiher, M.; Salomon, O.; Hess, B. A. Theor. Chem. Acc. 2001, 107, 48-55.

d. Zhao, Y.; Truhlar, D. G. Theor. Chem. Acc. 2008, 120, 215-241.

e. Zhao, Y.; Truhlar, D. G. J. Chem. Phys. 2006, 125, 194101: 1-18.

f. $\Delta G^{\ddagger}$ refers to the overall barrier of the reaction. 
Table S2. Calculated free energies ( $\mathrm{kcal} / \mathrm{mol}$ ) relative to $\boldsymbol{C} \cdot \mathbf{4}$ for hydrolysis of HPNP.

\begin{tabular}{ccccccccc}
\hline Method & C·4B & 4-TS1 & C·4Int & 4-TS2 & C·5·6 & C·5 & $\begin{array}{c}\text { Reaction } \\
(2)\end{array}$ & $\boldsymbol{\Delta G}^{*}$ \\
\hline B3LYP-D3 & 7.9 & 16.4 & 13.2 & 10.8 & -9.0 & -9.2 & -6.1 & $\mathbf{1 9 . 5}$ \\
B3LYP-D3 (RRHO) $^{\mathrm{a}}$ & 7.5 & 16.2 & 13.2 & 11.4 & -10.3 & -7.3 & -4.6 & $\mathbf{2 1 . 9}$ \\
B3LYP $^{\mathrm{b}}$ & 1.3 & 13.2 & 12.4 & 11.4 & -12.8 & -24.2 & -12.6 & $\mathbf{2 4 . 8}$ \\
B3LYP*-D3 $^{\mathrm{c}}$ & 8.4 & 15.6 & 12.5 & 10.2 & -8.3 & -7.7 & -5.8 & $\mathbf{1 8 . 1}$ \\
M06 $^{\mathrm{d}}$ & 8.5 & 19.9 & 16.7 & 13.5 & -10.2 & -14.0 & -7.3 & $\mathbf{2 6 . 6}$ \\
M06-D3 & 9.7 & 20.5 & 16.7 & 13.0 & -10.0 & -9.9 & -6.1 & $\mathbf{2 4 . 4}$ \\
M06-L & 10.2 & 19.6 & 16.1 & 13.4 & -10.8 & -15.8 & -11.5 & $\mathbf{2 3 . 9}$ \\
\hline
\end{tabular}

a. Entropies calculated using standard rigid-rotor harmonic oscillator approximation, i.e. without special treatment of vibrational modes with low wavenumbers.

b. Calculated with standard B3LYP functional, i.e.not including the DFT-D3 dispersion correction. c. $15 \%$ Hartree-Fock exchange is used, as has been suggested for complexes containing transition metals, see Reiher, M.; Salomon, O.; Hess, B. A. Theor. Chem. Acc. 2001, 107, 48-55.

d. Zhao, Y.; Truhlar, D. G. Theor. Chem. Acc. 2008, 120, 215-241.

e. Zhao, Y.; Truhlar, D. G. J. Chem. Phys. 2006, 125, 194101: 1-18.

f. $\Delta G^{\ddagger}$ refers to the overall barrier of the reaction. 


\section{S1.B Results with different solvation models}

The results with two solvation models (C-PCM and SMD) are presented in Table S3 for BDNPP and Table S4 for HPNP. For each solvation model, the calculations were done with water and acetonitrile as solvent.

The choice of solvation model affects mainly the stability of the charged catalyst-product complexes $\mathbf{C} \cdot \mathbf{2}^{+}$and $\mathbf{C} \cdot \mathbf{5}^{+}$, relative to the corresponding catalyst-substrate complexes. The largest difference is found for the hydrolysis of BDNPP for which C-PCM/water resulted in a barrier ca $8 \mathrm{kcal} / \mathrm{mol}$ lower than with $\mathrm{SMD} /$ water $(21.0 \mathrm{vs}$. $29.3 \mathrm{kcal} / \mathrm{mol})$. The C$\mathrm{PCM}$ /acetonitrile and SMD/acetonitrile results, on the other hand, are rather similar (22.7 vs. $21.4 \mathrm{kcal} / \mathrm{mol}$ ).

The overall barrier for transesterification of HPNP was calculated to be of similar height regardless of the solvation model employed.

Table S3. Calculated free energies $(\mathrm{kcal} / \mathrm{mol})$ relative to $\boldsymbol{C} \cdot \mathbf{1}$ for hydrolysis of BDNPP.

\begin{tabular}{cccccccccc}
\hline $\begin{array}{c}\text { Solvation } \\
\text { model }\end{array}$ & Solvent & $\mathbf{C} \cdot \mathbf{1 B}$ & $\mathbf{1 - T S 1}$ & $\mathbf{C} \cdot \mathbf{1}$ Int & $\mathbf{1 - T S 2}$ & $\mathbf{C} \cdot \mathbf{2} \cdot \mathbf{3}$ & $\mathbf{C}^{+} \mathbf{2}^{+}$ & $\begin{array}{c}\text { Reaction } \\
(\mathbf{1})\end{array}$ & $\boldsymbol{\Delta} \boldsymbol{G}^{\ddagger \mathrm{c}}$ \\
\hline $\mathrm{C}-\mathrm{PCM}^{\mathrm{a}}$ & $\mathrm{H}_{2} \mathrm{O}$ & 7.2 & 10.8 & 9.4 & 8.6 & -20.2 & -18.4 & -10.0 & $\mathbf{2 1 . 0}$ \\
$\mathrm{C}-\mathrm{PCM}^{\mathrm{a}}$ & $\mathrm{CH}_{3} \mathrm{CN}$ & 7.7 & 12.5 & 11.3 & 10.5 & -19.8 & -16.7 & -9.6 & $\mathbf{2 2 . 7}$ \\
$\mathrm{SMD}^{\mathrm{b}}$ & $\mathrm{H}_{2} \mathrm{O}$ & 5.5 & 11.9 & 9.5 & 2.5 & -18.4 & -26.8 & -9.4 & $\mathbf{2 9 . 3}$ \\
$\mathrm{SMD}^{\mathrm{b}}$ & $\mathrm{CH}_{3} \mathrm{CN}$ & 7.8 & 11.3 & 10.0 & 7.8 & -19.0 & -20.0 & -9.9 & $\mathbf{2 1 . 4}$ \\
\hline
\end{tabular}

a. Cossi, M.; Rega, N.; Scalmani, G.; Barone, V. J. Comp. Chem. 2003, 24, 669-681.

b. Marenich, A. V.; Cramer, C. J.; Truhlar, D. G. J. Phys. Chem. B 2009, 113, 6378-6396.

c. $\Delta G^{\ddagger}$ refers to the overall barrier of the reaction.

Table S4. Calculated free energies $(\mathrm{kcal} / \mathrm{mol})$ relative to $\boldsymbol{C} \cdot 4$ for hydrolysis of HPNP.

\begin{tabular}{|c|c|c|c|c|c|c|c|c|c|}
\hline $\begin{array}{c}\text { Solvation } \\
\text { model }\end{array}$ & Solvent & $C \cdot 4 B$ & 4-TS1 & $C \cdot 4$ Int & 4-TS2 & $C \cdot 5 \cdot 6$ & $C \cdot 5^{+}$ & $\begin{array}{c}\text { Reaction } \\
\text { (2) }\end{array}$ & $\Delta G^{\ddagger c}$ \\
\hline C-PCM ${ }^{\mathrm{a}}$ & $\mathrm{H}_{2} \mathrm{O}$ & 7.9 & 16.4 & 13.2 & 10.8 & -9.0 & -9.2 & -6.1 & 19.5 \\
\hline C-PCM ${ }^{\mathrm{a}}$ & $\mathrm{CH}_{3} \mathrm{CN}$ & 8.9 & 17.5 & 13.8 & 11.2 & -8.1 & -7.7 & -5.6 & 20.0 \\
\hline $\mathrm{SMD}^{\mathrm{b}}$ & $\mathrm{H}_{2} \mathrm{O}$ & 1.2 & 9.1 & 4.7 & 0.1 & -10.3 & -16.1 & -6.3 & 18.9 \\
\hline $\mathrm{SMD}^{\mathrm{b}}$ & $\mathrm{CH}_{3} \mathrm{CN}$ & 6.4 & 14.4 & 11.1 & 8.3 & -9.3 & -8.0 & -1.6 & 22.1 \\
\hline
\end{tabular}

a. Cossi, M.; Rega, N.; Scalmani, G.; Barone, V. J. Comp. Chem. 2003, 24, 669-681.

b. Marenich, A. V.; Cramer, C. J.; Truhlar, D. G. J. Phys. Chem. B 2009, 113, 6378-6396.

c. $\Delta G^{\sharp}$ refers to the overall barrier of the reaction. 


\section{S2. Hydrolysis of BDNPP with two hydroxides}

The free energy profile for hydrolysis of BDNPP carried out with two hydroxides bound to the catalyst throughout the reaction is shown in Figure S1.

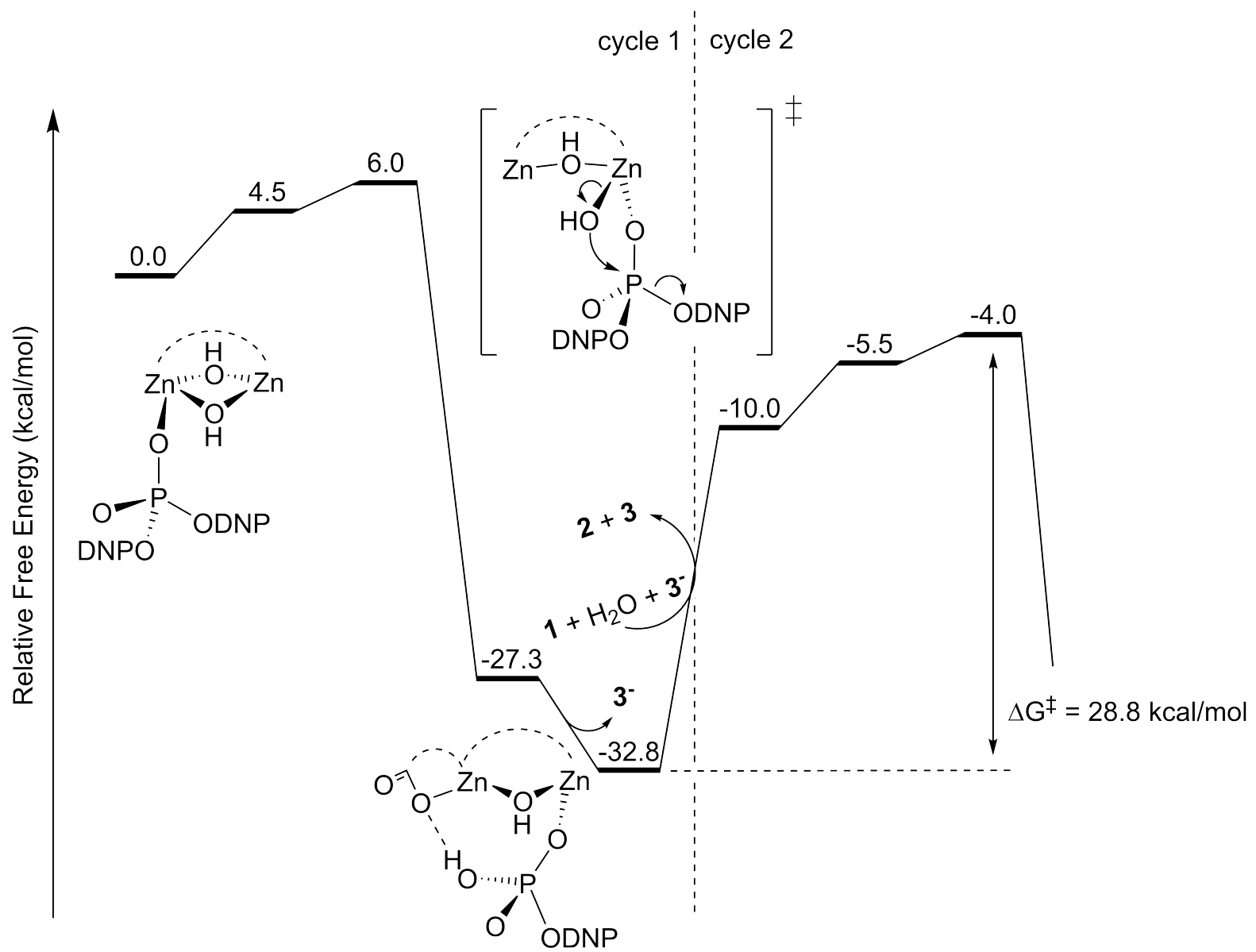

Figure S1. Free energy profile for hydrolysis of BDNPP with two hydroxides bound to the catalyst.

In the lowest-energy catalyst-substrate complex it is found that the substrate coordinates to $\mathrm{Zn} 1$, while both hydroxides are bridging the zinc ions. However, in the transition state with lowest energy, the substrate binds to $\mathrm{Zn} 2$ via a phosphate oxygen, and a hydroxide that is coordinated terminally to the same zinc ion attacks the phosphorus center of the substrate. The catalyst-product complex, in which only the phosphate product is bound to the catalyst, was calculated to be the resting state of the catalytic cycle. The calculated overall barrier is 28.8 $\mathrm{kcal} / \mathrm{mol}$, which is $7.8 \mathrm{kcal} / \mathrm{mol}$ higher than what was found with only one hydroxide bound to the catalyst $(21.0 \mathrm{kcal} / \mathrm{mol})$. 


\section{S3. Results with the DPCPMP ligand}

As discussed in the paper, the hydrolysis of BDNPP was recently studied with the DPCPMP ligand [1]. Here, we have re-done these calculations according to the computational protocol used in the present paper. The methodology applied here differs from the one in the previous study in two aspects: the treatment of vibrational modes with low frequencies and the inclusion of standard state corrections. The resulting free energy profile is given in Figure S2 and is compared with the one obtained with the IPCPMP ligand. The overall barrier is calculated to be $25.0 \mathrm{kcal} / \mathrm{mol}$, in good agreement with the experimental barrier of ca. 26 $\mathrm{kcal} / \mathrm{mol}[1]$. The value reported previously was $30.6 \mathrm{kcal} / \mathrm{mol}$.

It should also be mentioned that a slightly new structure for the catalyst-substrate complex could be located that is slightly lower in energy compared to the previous report [1] and that is more analogous to the one found for the IPCPMP ligand (see Figure S3).

Furthermore, in the previous study [1] the reaction mechanism was denoted as concerted without mentioning of the details of leaving group dissociation. Similarly to the IPCPMP case, the transition state for dissociation of the leaving group with the DPCPMP ligand (Figure S3) was calculated to be lower in energy than the pentacoordinate intermediate $(9.8$ vs. $11.0 \mathrm{kcal} / \mathrm{mol}$ ), making the reaction effectively concerted.

Finally, in the previous study, the transition state for the general base mechanism (corresponding to Figure 5A in the paper for IPCPMP) was not located. It has now been done and the transition state is shown in Figure S3. The barrier is calculated to be $13.9 \mathrm{kcal} / \mathrm{mol}$ relative to $\left(\mathbf{C} \cdot \mathbf{1}+\mathrm{H}_{2} \mathrm{O}\right)$, i.e. $2.2 \mathrm{kcal} / \mathrm{mol}$ higher than the transition state for the nucleophilic attack mechanism. The corresponding difference for the IPCPMP complex is $6.4 \mathrm{kcal} / \mathrm{mol}$. These results suggest thus that for the DPCPMP complex such a general-base mechanism cannot be ruled out on the basis of the calculations.

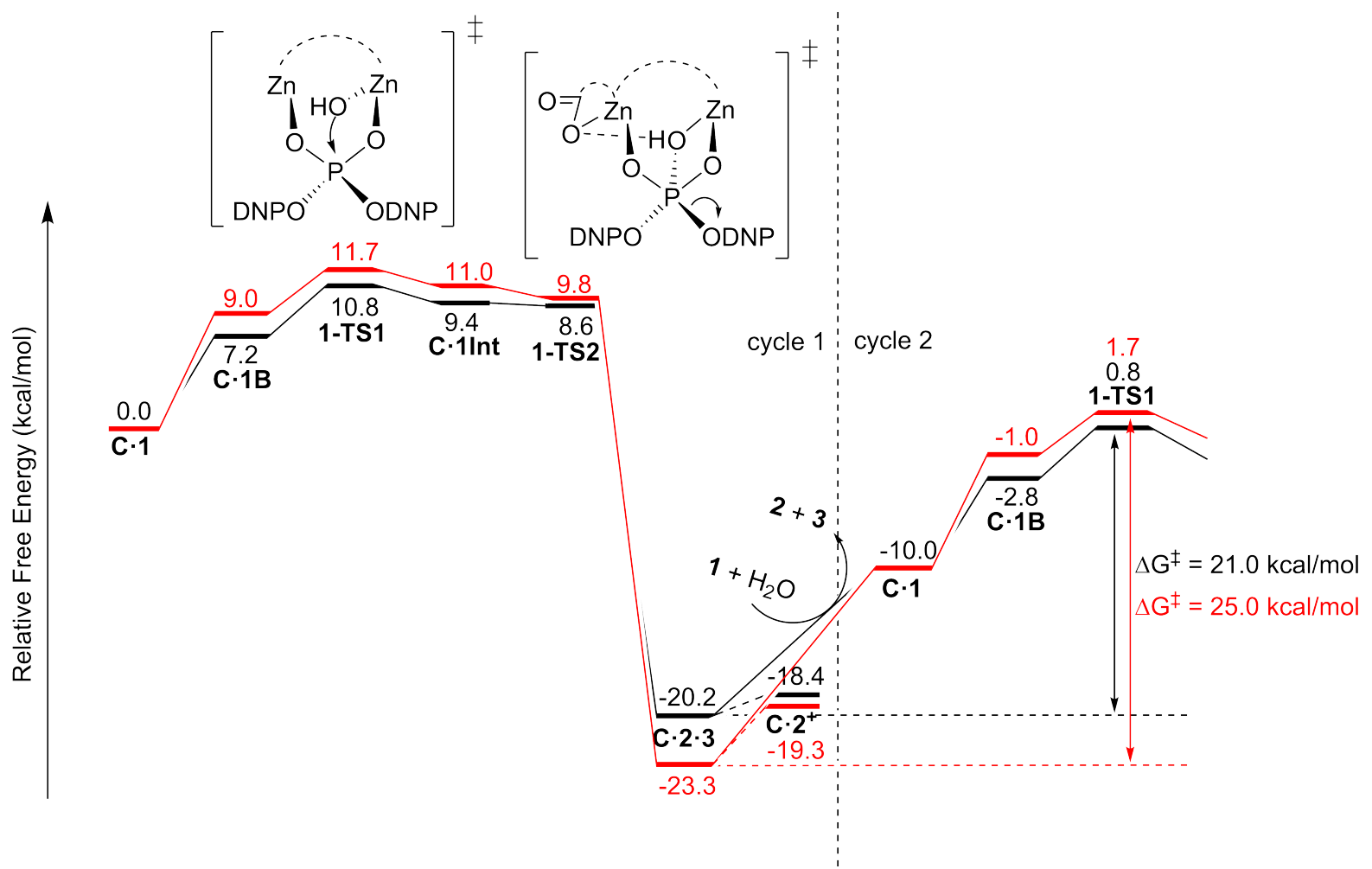

Figure S2. Free energy profile for hydrolysis of BDNPP with the DPCPMP ligand (red) compared to the IPCPMP ligand (black). 

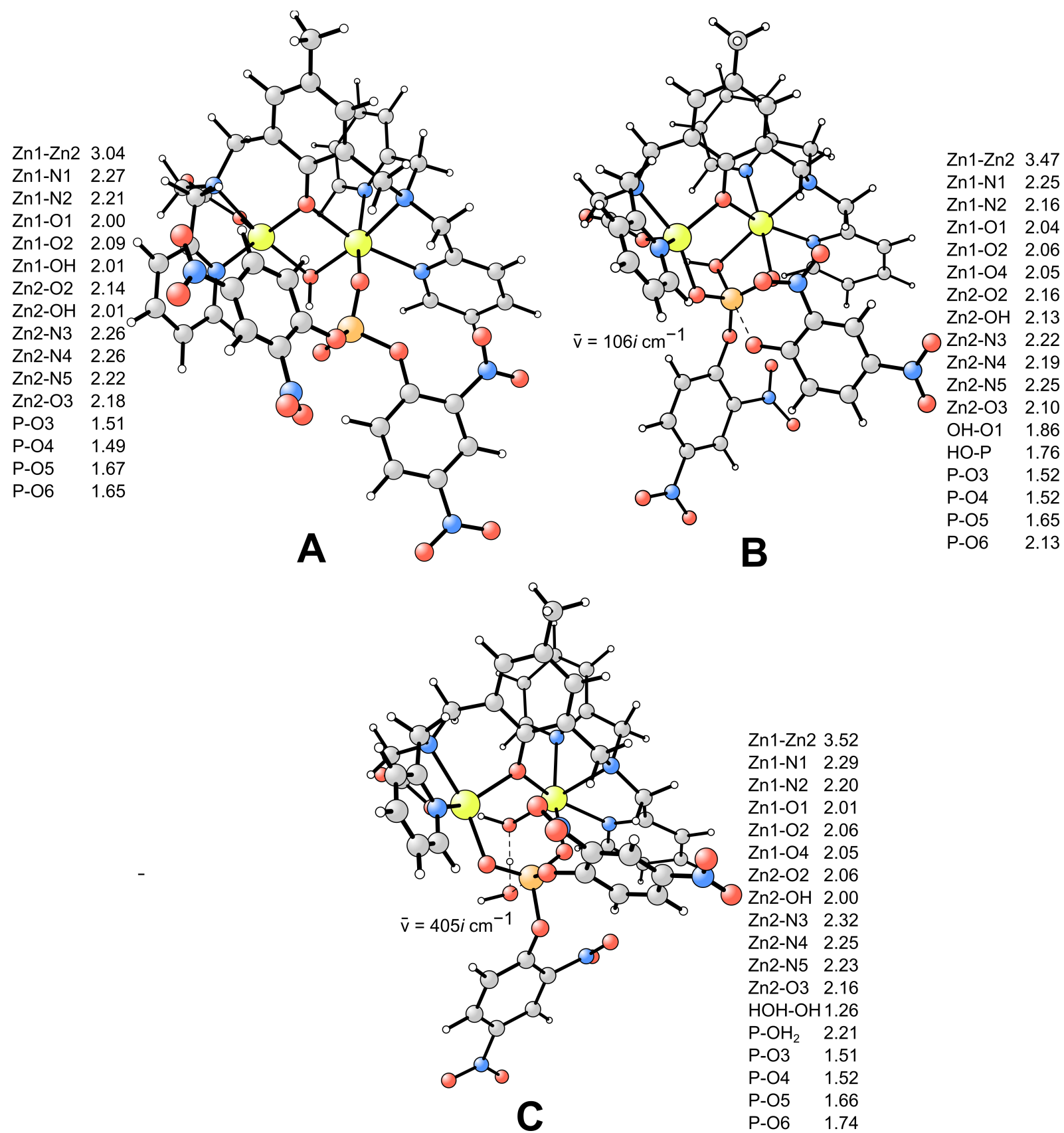

Figure S3. Optimized structures of: A) A slightly new structure for the catalyst-substrate complex with the DPCPMP ligand, B) Transition state for dissociation of the leaving group, and $C)$ Transition state for the general base mechanism.

[1] Das, B.; Daver, H.; Pyrkosz-Bulska, M.; Persch, E.; Barman, S. K.; Mukherjee, R.; Gumienna-Kontecka, E.; Jarenmark, M.; Himo, F.; Nordlander, E. J. Inorg. Biochem. 2014, $132,6-17$. 


\section{S4. Alternative mechanisms with HPNP}

When two hydroxides are bound to the catalyst throughout the reaction, one hydroxide is found to bridge the zinc ions while the other is coordinated terminally to $\mathrm{Zn} 2$ in the lowestenergy catalyst-substrate complex. In the calculations, it was found that the terminal hydroxide must coordinate to $\mathrm{Zn} 1$ in order for the reaction to proceed.

The free energy profile for transesterification of HPNP with two hydroxides is shown in Figure S4. Optimized transition states for deprotonation of the substrate and for the subsequent nucleophilic attack are given in Figure S5. The overall barrier for the reaction is calculated to be $33.1 \mathrm{kcal} / \mathrm{mol}$, i.e. $13.6 \mathrm{kcal} / \mathrm{mol}$ higher than with one hydroxide. Interestingly, a similar overall barrier $(32.3 \mathrm{kcal} / \mathrm{mol})$ was obtained for hydrolysis of HPNP, when the nucleophile is the hydroxide that is bound to $\mathrm{Zn} 1$. The free energy profile for this reaction is shown in Figure S6.

To investigate the feasibility of a mechanism for transesterification of HPNP that involves a pre-equilibrium, where the substrate is deprotonated by the solvent, the catalytic cycle was calculated with the deprotonated substrate bound to the catalyst. The resulting free energy profile is shown in Figure S7. The overall barrier is calculated to be $29.3 \mathrm{kcal} / \mathrm{mol}$, i.e. 9.8 $\mathrm{kcal} / \mathrm{mol}$ higher than when deprotonation of HPNP takes place in concert with the nucleophilic attack.

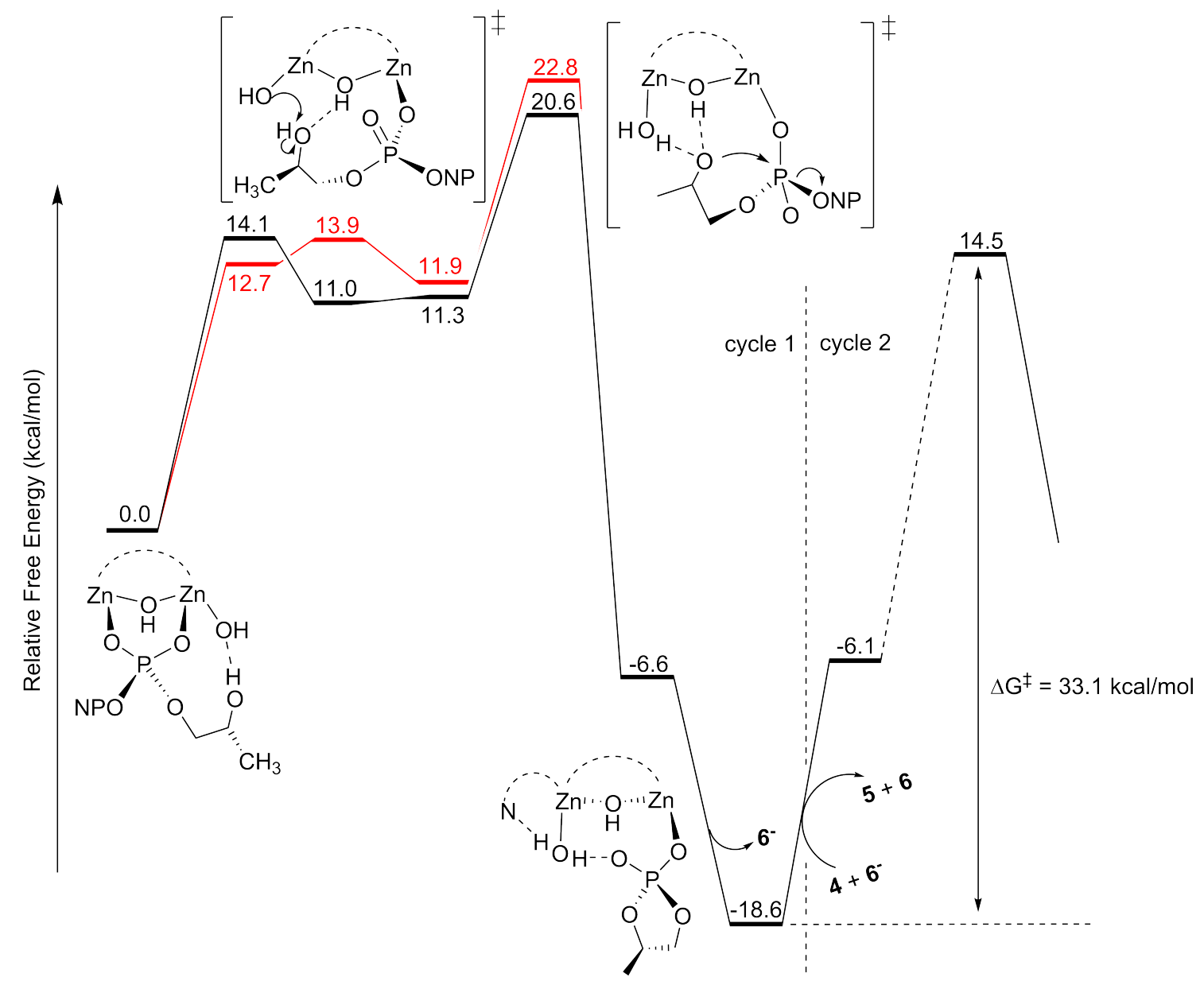

Figure S4. Free energy profile for transesterification of HPNP with two hydroxides. The transition state for deprotonation of the substrate is stabilized by solvation compared to the preceding and following intermediates; gas-phase values are shown in red. 
Zn1-Zn2 3.24

Zn1-N1 2.46

$\mathrm{Zn} 1-\mathrm{N} 2 \quad 2.28$

Zn1-O1 2.05

$\mathrm{Zn} 1-\mathrm{O} 2 \quad 2.52$

$\mathrm{Zn} 1-\mathrm{O}_{\mathrm{b}} \mathrm{H} 2.01$

$\mathrm{Zn} 1-\mathrm{OH} 2.16$

$\mathrm{Zn} 2-\mathrm{O} 2 \quad 2.02$

$\mathrm{Zn} 2-\mathrm{O}_{\mathrm{b}} \mathrm{H} 1.96$

Zn2-N3 2.42

Zn2-N4 2.20

Zn2-O3 2.05

$\mathrm{O}_{\mathrm{b}} \mathrm{H}-\mathrm{O} 7 \quad 1.76$

HO-HO7 1.14

$\mathrm{P}-\mathrm{O} 3 \quad 1.53$

$\mathrm{P}-\mathrm{O} 4 \quad 1.48$

P-O5 1.61

P-O6 1.69

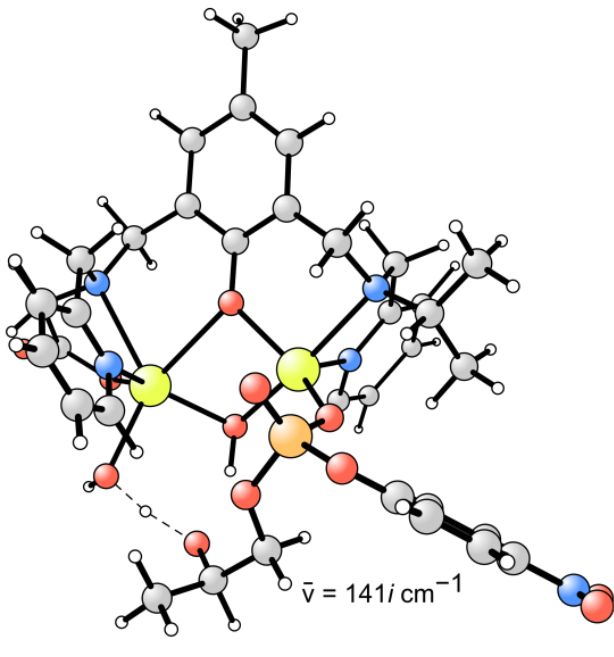

A

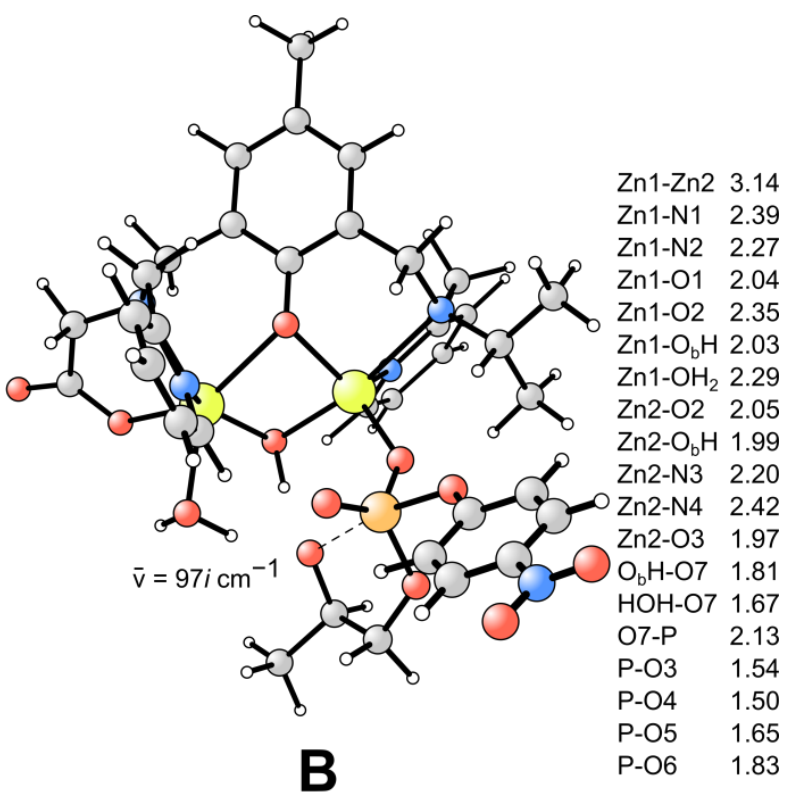

B

Figure S5. Optimized transition states for transesterification of HPNP with two hydroxides. A) Deprotonation of HPNP. B) Nucleophilic attack.

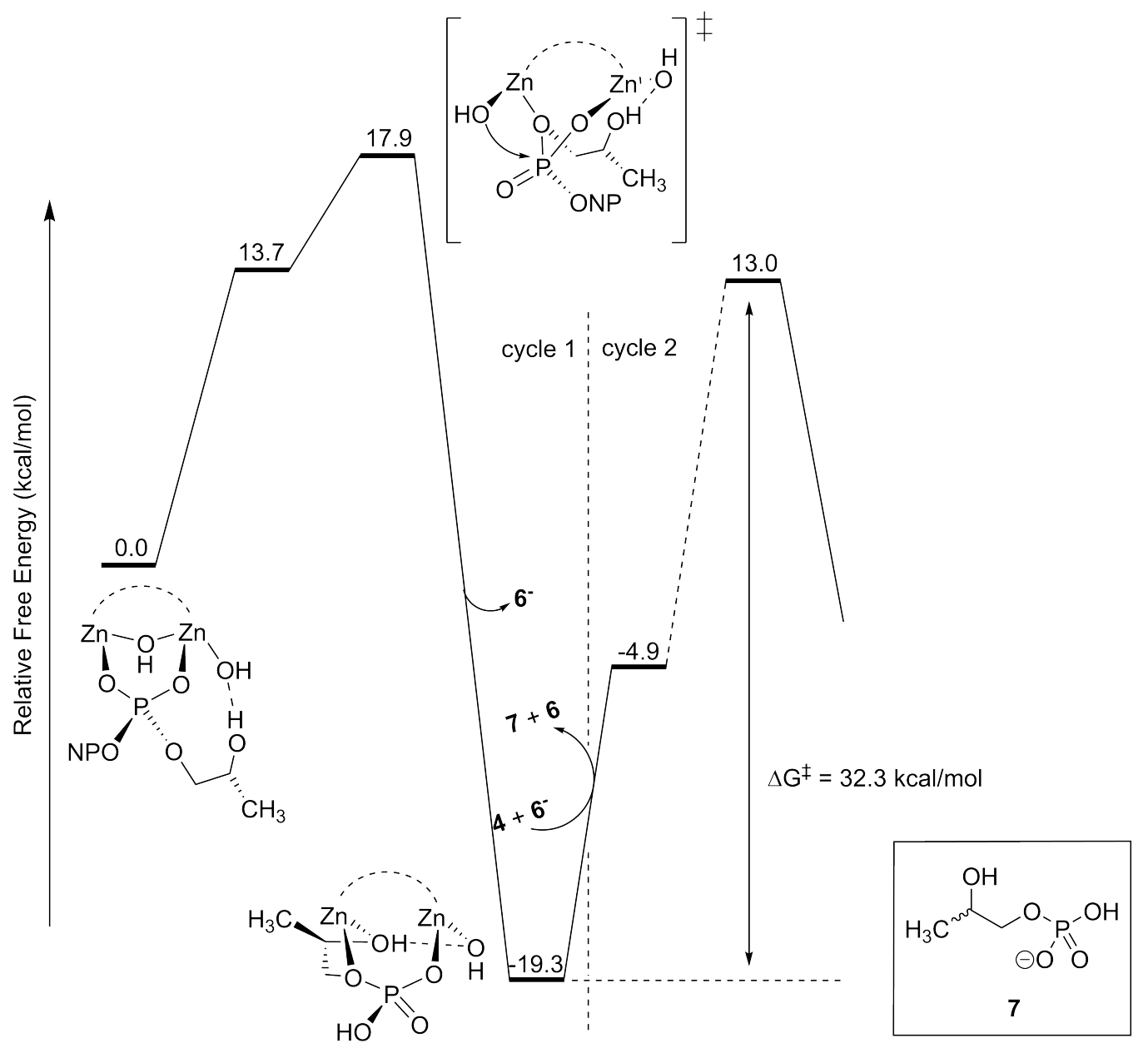

Figure S6. Free energy profile for hydrolysis of HPNP with two hydroxides. 


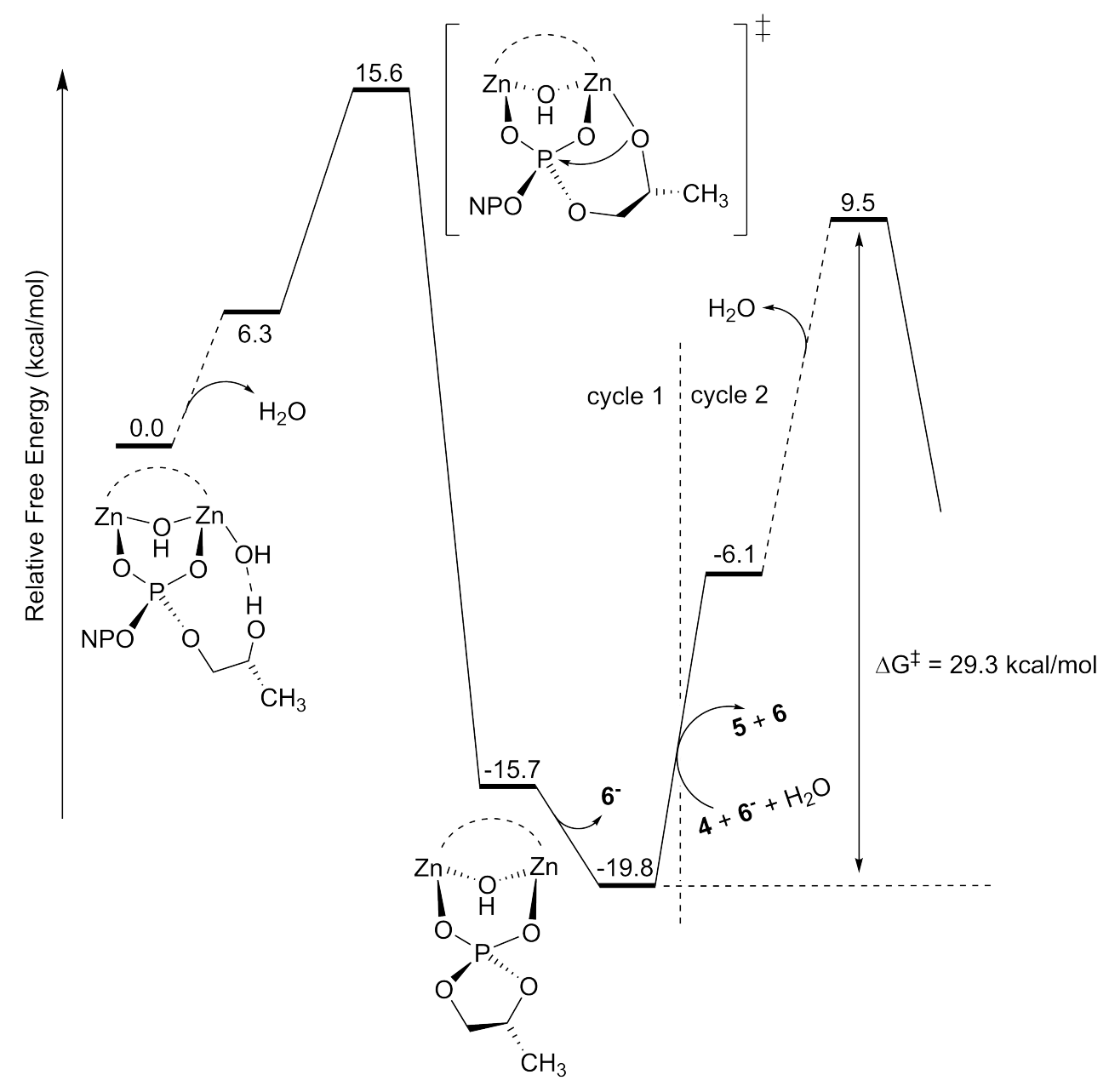

Figure S7. Free energy profile for transesterification of HPNP assuming that the substrate is in the deprotonated form. 


\section{S5. Calculated absolute energies and energy corrections}

Table S5. Calculated absolute energies and energy corrections (in atomic units)

\begin{tabular}{lcccc}
\hline & $\begin{array}{c}\text { Absolute energy } \\
\text { large basis set }\end{array}$ & $\begin{array}{c}\text { Solvation } \\
\text { energy }\end{array}$ & $\begin{array}{c}\text { Thermal correction } \\
\text { to Gibbs free energy }\end{array}$ & $\begin{array}{c}\text { Dispersion } \\
\text { correction }\end{array}$ \\
\hline $\mathrm{H}_{2} \mathrm{O}$ & -76.461926 & -0.010177 & 0.003715 & -0.000574 \\
$\mathbf{1}$ & -1924.294565 & -0.072150 & 0.155087 & -0.086091 \\
$\mathbf{2}$ & -1284.045829 & -0.084142 & 0.080916 & -0.046517 \\
$\mathbf{3}$ & -716.712035 & -0.012505 & 0.074930 & -0.033578 \\
$\mathbf{3}^{-}$ & -716.193829 & -0.080607 & 0.060828 & -0.032035 \\
$\mathbf{4}$ & -1272.706139 & -0.081415 & 0.167667 & -0.060118 \\
$\mathbf{5}$ & -760.521248 & -0.105408 & 0.068771 & -0.022831 \\
$\mathbf{6}$ & -512.141817 & -0.018397 & 0.075112 & -0.026925 \\
$\mathbf{4}^{-}$ & -1272.014189 & -0.260279 & 0.149309 & -0.055399 \\
$\mathbf{6}$ & -511.615303 & -0.085961 & 0.062072 & -0.025820 \\
$\mathbf{C} \cdot \mathbf{1}$ & -3585.417274 & -0.059554 & 0.665175 & -0.294859 \\
$\mathbf{C} \cdot \mathbf{1 B}$ & -3585.407881 & -0.055623 & 0.666622 & -0.298197 \\
$\mathbf{1 - T S 1}$ & -3585.404369 & -0.056940 & 0.667983 & -0.295933 \\
$\mathbf{C} \cdot \mathbf{1}$ Int & -3585.404402 & -0.059174 & 0.668026 & -0.295915 \\
$\mathbf{1 - T S 2}$ & -3585.397097 & -0.070293 & 0.666800 & -0.292293 \\
$\mathbf{C} \cdot \mathbf{2} \cdot \mathbf{3}$ & -3585.456094 & -0.052543 & 0.667742 & -0.297841 \\
$\mathbf{C} \cdot \mathbf{2}^{+}$ & -2869.140925 & -0.088844 & 0.579925 & -0.243433 \\
$\mathbf{1 - T S 1 ( b a s e )}$ & -3661.871307 & -0.055872 & 0.689716 & -0.303741 \\
$\mathbf{C} \cdot \mathbf{4}$ & -2933.851833 & -0.054127 & 0.676950 & -0.272699 \\
$\mathbf{C} \cdot \mathbf{4 B}$ & -2933.847999 & -0.056080 & 0.677103 & -0.262106 \\
$\mathbf{4 - T S 1}$ & -2933.830200 & -0.052596 & 0.674754 & -0.267611 \\
$\mathbf{C} \cdot \mathbf{4 I n t}$ & -2933.836445 & -0.051919 & 0.679122 & -0.271468 \\
$\mathbf{4 - T S 2}$ & -2933.835451 & -0.054764 & 0.679348 & -0.273627 \\
$\mathbf{C} \cdot \mathbf{5} \cdot \mathbf{6}$ & -2933.866132 & -0.058296 & 0.674963 & -0.266536 \\
$\mathbf{C} \cdot \mathbf{5}^{+}$ & -2422.143255 & -0.078607 & 0.590459 & -0.222942 \\
$\mathbf{4 - T S 1 ( b a s e )}$ & -3010.298628 & -0.055620 & 0.696703 & -0.274516 \\
\hline & & & &
\end{tabular}




\section{S6. Cartesian coordinates}

1

$\begin{array}{rrrr}\text { C } & 3.249471 & 1.289696 & 0.639557 \\ \mathrm{C} & 2.276851 & 0.446206 & 1.146402 \\ \mathrm{C} & 1.914964 & -0.737889 & 0.463574 \\ \mathrm{C} & 2.585489 & -1.020566 & -0.756498 \\ \mathrm{C} & 3.542425 & -0.161939 & -1.282358 \\ \mathrm{C} & 3.878212 & 0.982516 & -0.572327 \\ \mathrm{H} & 3.529002 & 2.193254 & 1.166817 \\ \mathrm{H} & 1.783103 & 0.668942 & 2.083658 \\ \mathrm{H} & 4.015005 & -0.389675 & -2.227673 \\ \mathrm{C} & -3.391074 & -1.148236 & -0.786474 \\ \mathrm{C} & -3.986552 & 0.089806 & -1.053072 \\ \mathrm{C} & -3.561147 & 1.242416 & -0.406254 \\ \mathrm{C} & -2.509588 & 1.153910 & 0.496207 \\ \mathrm{C} & -1.892428 & -0.086529 & 0.810820 \\ \mathrm{C} & -2.366693 & -1.237879 & 0.139813 \\ \mathrm{H} & -3.750019 & -2.029382 & -1.303769 \\ \mathrm{H} & -4.030832 & 2.196173 & -0.603296 \\ \mathrm{H} & -1.925317 & -2.193594 & 0.395483 \\ \mathrm{O} & -0.939574 & -0.102929 & 1.737301 \\ \mathrm{O} & 0.955707 & -1.566644 & 0.869758 \\ \mathrm{P} & -0.008522 & -1.439339 & 2.275240 \\ \mathrm{O} & 0.796183 & -0.886355 & 3.390536 \\ \mathrm{O} & -0.841682 & -2.665659 & 2.258284 \\ \mathrm{~N} & 2.305488 & -2.234445 & -1.534453 \\ \mathrm{~N} & 4.901943 & 1.873761 & -1.105152 \\ \mathrm{~N} & -5.075113 & 0.178384 & -2.019059 \\ \mathrm{~N} & -2.066854 & 2.414877 & 1.104909 \\ \mathrm{O} & 2.420069 & -2.147758 & -2.761332 \\ \mathrm{O} & 2.012083 & -3.262988 & -0.933397 \\ \mathrm{O} & -0.871421 & 2.564833 & 1.339333 \\ \mathrm{O} & -2.933662 & 3.269929 & 1.307329 \\ \mathrm{O} & 5.435857 & 1.569201 & -2.175355 \\ \mathrm{O} & -5.572341 & 1.287634 & -2.231673 \\ \mathrm{O} & 5.180522 & 2.884403 & -0.454623 \\ \mathrm{O} & -5.440606 & -0.862764 & -2.571145\end{array}$

2
$0-4.106523-2.083832-0.007956$

4

$\begin{array}{llll}\text { C } & 2.636358 & 0.690643 & 0.775800\end{array}$

$\begin{array}{llll}\text { C } \quad 3.453592 & -0.169872 & 0.034754\end{array}$

C $\quad 2.949602-0.863089-1.072385$

$\begin{array}{llll}\text { C } & 1.623170 & -0.693634 & -1.427700\end{array}$

$\begin{array}{llll}\text { C } & 0.780888 & 0.167022 & -0.688259\end{array}$

$\begin{array}{llll}\text { C } & 1.306814 & 0.865298 & 0.421254\end{array}$

$\mathrm{H} \quad 3.059688 \quad 1.218276 \quad 1.621754$

$\mathrm{H} \quad 3.605878-1.520714-1.628799$

$\mathrm{H} \quad 1.199144-1.216853-2.278318$

$\begin{array}{llll}\mathrm{H} & 0.664283 & 1.548486 & 0.963344\end{array}$

$\begin{array}{llll}\mathrm{N} & 4.841765 & -0.346184 & 0.413901\end{array}$

$\begin{array}{llll}0 & 5.542925 & -1.109439 & -0.264332\end{array}$

$\begin{array}{llll}0 & 5.265825 & 0.272446 & 1.398226\end{array}$

$\begin{array}{llll}0 & -0.488321 & 0.253042 & -1.110570\end{array}$

$\begin{array}{llll}\mathrm{P}-1.768718 & 1.120836 & -0.360785\end{array}$

$\begin{array}{llll}0 & -2.904044 & 0.936068 & -1.324588\end{array}$

$\begin{array}{llll}0 & -1.981338 & 0.185635 & 0.975232\end{array}$

$\begin{array}{llll}0 & -1.259852 & 2.441237 & 0.104899\end{array}$

$\begin{array}{lll}\text { C }-2.365565 & -1.194559 & 0.915818\end{array}$

$\begin{array}{llll}\text { C }-3.843152 & -1.467427 & 0.573943\end{array}$

$\begin{array}{llll}0 & -4.112815 & -1.404960 & -0.811344\end{array}$

$\mathrm{H}-3.766766-0.537165-1.133559$

$\begin{array}{llll}\mathrm{H} & -1.737933 & -1.731823 & 0.193332\end{array}$

$\mathrm{H}-2.152288-1.586157 \quad 1.917728$

$\begin{array}{llll}\text { C }-4.804901 & -0.589249 & 1.385207\end{array}$

$\mathrm{H}-5.837205-0.867985 \quad 1.152381$

$\mathrm{H}-4.644250-0.704017 \quad 2.464396$

$\begin{array}{llll}\mathrm{H} & -4.664955 & 0.464558 & 1.130051\end{array}$

H $-4.007052-2.518892 \quad 0.861385$

\section{5}

$\begin{array}{llll}\mathrm{P}-1.110762 & -0.136708 & 0.032352\end{array}$

$\begin{array}{llll}0 & -1.929466 & -0.389227 & -1.193397\end{array}$

$\begin{array}{llll}0 & -0.351681 & 1.380578 & -0.141179\end{array}$

$\begin{array}{llll}0 & -1.637671 & -0.296541 & 1.424270\end{array}$

$\begin{array}{llll}\text { C } & 1.033730 & 1.275325 & 0.107252\end{array}$

C $1.448266-0.111373-0.417922$

$\begin{array}{llll}\text { C } & 2.753949 & -0.635187 & 0.162243\end{array}$

O $0.378076-0.968493-0.062177$

$\mathrm{H} \quad 2.970508-1.636105-0.223189$

$\mathrm{H} \quad 2.684821-0.696081 \quad 1.253216$

$\begin{array}{lllll}\mathrm{H} & 3.589727 & 0.024880 & -0.103984\end{array}$

H $\quad 1.524769-0.061108-1.518594$

H $1.245753 \quad 1.346362 \quad 1.188143$

H $\quad 1.556116 \quad 2.089547-0.410447$

$4^{-}$

$\begin{array}{lrrr}\text { C }-2.437558 & 0.187428 & 1.033486\end{array}$

$\begin{array}{llll}\text { C }-3.361881 & 0.303690 & -0.019447\end{array}$

C $-3.004665-0.106845-1.315101$

C $-1.746382-0.629303-1.541896$

C $-0.801852-0.766190-0.489682$

C $-1.176279-0.337497 \quad 0.810969$

$\begin{array}{llll}\mathrm{H} & -2.735702 & 0.513357 & 2.022878\end{array}$

$\mathrm{H}-3.728730-0.004286-2.114411$

H $-1.444973-0.952174-2.533565$ 


$\begin{array}{rrrr}\mathrm{H} & -0.462284 & -0.458792 & 1.616634 \\ \mathrm{~N} & -4.670579 & 0.832747 & 0.223209 \\ \mathrm{O} & -5.472087 & 0.919507 & -0.733993 \\ \mathrm{O} & -4.973869 & 1.186821 & 1.382447 \\ \mathrm{O} & 0.366621 & -1.299762 & -0.807908 \\ \mathrm{P} & 1.750199 & -1.596894 & 0.255764 \\ \mathrm{O} & 2.631437 & -2.427566 & -0.622659 \\ \mathrm{O} & 2.272591 & -0.079944 & 0.444336 \\ \mathrm{O} & 1.179295 & -2.065242 & 1.563481 \\ \mathrm{C} & 3.042593 & 0.614610 & -0.591732 \\ \mathrm{C} & 3.528164 & 2.027955 & -0.159570 \\ \mathrm{O} & 4.151976 & 2.618827 & -1.180566 \\ \mathrm{H} & 3.911431 & 0.000508 & -0.853880 \\ \mathrm{H} & 2.413783 & 0.734196 & -1.481386 \\ \mathrm{C} & 4.409928 & 1.911058 & 1.124639 \\ \mathrm{H} & 5.321354 & 1.346021 & 0.880256 \\ \mathrm{H} & 4.721966 & 2.918676 & 1.431347 \\ \mathrm{H} & 3.902523 & 1.418850 & 1.966732 \\ \mathrm{H} & 2.581581 & 2.557258 & 0.195373\end{array}$

\section{$C \cdot 1$}

$\begin{array}{rrrr}\text { Zn } & 0.218631 & -1.431379 & 0.275969 \\ \mathrm{Zn} & 1.869306 & -0.363975 & -2.093201 \\ \mathrm{O} & 2.409133 & -1.314688 & -3.746271 \\ \mathrm{O} & 2.200398 & -0.995749 & -0.098301 \\ \mathrm{O} & 4.012924 & -1.469192 & -5.329800 \\ \mathrm{~N} & 1.939610 & 1.868326 & -2.162131 \\ \mathrm{~N} & 4.081344 & 0.152265 & -2.112545 \\ \mathrm{~N} & 0.797257 & -2.314500 & 2.302951 \\ \mathrm{~N} & 0.122633 & -3.550022 & -0.111404 \\ \mathrm{C} & 0.896574 & 2.659325 & -2.465538 \\ \mathrm{H} & -0.049420 & 2.156645 & -2.645604 \\ \mathrm{C} & 1.005652 & 4.044606 & -2.536988 \\ \mathrm{H} & 0.141035 & 4.645090 & -2.796096 \\ \mathrm{C} & 2.245402 & 4.628675 & -2.272741 \\ \mathrm{H} & 2.365825 & 5.707072 & -2.305009 \\ \mathrm{C} & 3.326292 & 3.806504 & -1.962437 \\ \mathrm{H} & 4.306732 & 4.226562 & -1.761017 \\ \mathrm{C} & 3.141808 & 2.422071 & -1.914355 \\ \mathrm{C} & 4.279082 & 1.483286 & -1.539902 \\ \mathrm{H} & 4.300334 & 1.382612 & -0.447860 \\ \mathrm{H} & 5.239047 & 1.934711 & -1.836656 \\ \mathrm{C} & 4.365553 & 0.090995 & -3.563122 \\ \mathrm{H} & 4.081462 & 1.048065 & -4.014430 \\ \mathrm{H} & 5.434458 & -0.063316 & -3.759958 \\ \mathrm{C} & 3.548357 & -1.014260 & -4.294553 \\ \mathrm{C} & 4.806099 & -0.938060 & -1.412402 \\ \mathrm{H} & 4.481134 & -1.864204 & -1.902874 \\ \mathrm{H} & 5.886425 & -0.845235 & -1.605272 \\ \mathrm{C} & 4.591729 & -1.047761 & 0.081514 \\ \mathrm{C} & 5.717489 & -1.176944 & 0.901014 \\ \mathrm{H} & 6.701261 & -1.110856 & 0.439094 \\ \mathrm{C} & 5.629814 & -1.410681 & 2.275971 \\ \mathrm{C} & 6.865470 & -1.525120 & 3.137816 \\ \mathrm{H} & 6.680768 & -2.143909 & 4.021546 \\ \mathrm{H} & 7.699150 & -1.971306 & 2.586187 \\ \mathrm{H} & 7.203122 & -0.543789 & 3.495603 \\ \mathrm{C} & 4.347218 & -1.507108 & 2.819382 \\ \mathrm{H} & 4.240217 & -1.686954 & 3.887882 \\ \mathrm{C} & 3.185539 & -1.359059 & 2.051622 \\ \mathrm{C} & 3.294588 & -1.127273 & 0.655095\end{array}$

C $1.855638-1.350870$

$\mathrm{H} \quad 1.398051-0.357644$

H $2.067036-1.521915$

C $-0.368113-2.292038$

$\mathrm{H}-0.625085-1.232393$

C $-0.048582-2.823853$

$\mathrm{H} \quad 0.803878-2.327547$

$\mathrm{H}-0.915341-2.645387$

H $\quad 0.137380 \quad-3.903402$

C $-1.592292-3.024280$

$\mathrm{H}-1.418277-4.101486$

$\mathrm{H}-2.429860-2.890249$

$\mathrm{H}-1.907274-2.634723$

C $1.310461-3.671180$

H $2.380475-3.582525$

$\mathrm{H} \quad 1.229397-4.332011$

C $\quad 0.658284-4.343701$

C $0.680927-5.731317$

$\mathrm{H} \quad 1.110873-6.358219$

C $0.152288-6.288149-0.491840$

$\mathrm{H} \quad 0.162800 \quad-7.364737 \quad-0.634629$

C $-0.387511-5.450147-1.468403$

$\mathrm{H}-0.801332-5.845139-2.389259$

C $-0.384960-4.078232-1.238230$

$\mathrm{H}-0.783346-3.364112-1.951323$

$0-0.014869-0.806126-1.617728$

$\mathrm{H}-0.771470-0.235369-1.786260$

$\begin{array}{llll}\text { C }-0.886874 & 5.145117 & 0.336940\end{array}$

$\begin{array}{llll}\text { C }-1.783025 & 4.093642 & 0.468345\end{array}$

$\begin{array}{llll}\text { C }-1.570243 & 3.085746 & 1.419827\end{array}$

$\begin{array}{llll}\text { C }-0.441815 & 3.175477 & 2.258709\end{array}$

$\begin{array}{llll}\text { C } & 0.478596 & 4.208517 & 2.121254\end{array}$

C $0.241315 \quad 5.180414 \quad 1.158183$

$\mathrm{H}-1.035344 \quad 5.927349-0.396650$

$\mathrm{H}-2.651531 \quad 4.009357-0.174669$

H $\quad 1.361838 \quad 4.246757 \quad 2.744875$

$\begin{array}{llll}\text { C }-7.041652 & 0.676394 & 0.414115\end{array}$

C $-7.229861-0.046335-0.764304$

C $-6.209822-0.793143-1.336627$

$\begin{array}{llll}\text { C } & -4.973327 & -0.831195 & -0.700370\end{array}$

$\begin{array}{llll}\text { C }-4.742983 & -0.097748 & 0.481817\end{array}$

$\begin{array}{llll}\text { C }-5.794976 & 0.651148 & 1.024978\end{array}$

$\begin{array}{llll}\mathrm{H}-7.864726 & 1.247121 & 0.825613\end{array}$

$\mathrm{H}-6.370611-1.342142-2.254732$

$\mathrm{H}-5.600860 \quad 1.210492 \quad 1.933365$

$\begin{array}{llll}0 & -3.558690 & -0.105905 & 1.144075\end{array}$

$\begin{array}{llll}0 & -2.447687 & 2.057622 & 1.505842\end{array}$

$\begin{array}{llll}\mathrm{P} & -2.222454 & 0.682460 & 0.586203\end{array}$

$\begin{array}{llll}0 & -1.029669 & -0.041069 & 1.168488\end{array}$

$\begin{array}{lllll}0 & -2.335428 & 1.001477 & -0.863624\end{array}$

$\begin{array}{llll}\mathrm{N} & -0.175973 & 2.175699 & 3.303592\end{array}$

$\begin{array}{lrrr}\mathrm{N} & 1.227655 & 6.252372 & 0.976940\end{array}$

$\mathrm{N}-8.545459-0.019766-1.422086$

$\mathrm{N}-3.942091-1.684349-1.310691$

$\begin{array}{llll}0 & -1.130760 & 1.703776 & 3.910253\end{array}$

$\begin{array}{lrrr}0 & 1.006988 & 1.905554 & 3.517659\end{array}$

$\begin{array}{lllll}0 & -3.082190 & -2.167361 & -0.570617\end{array}$

$\begin{array}{llll}0 & -4.029483 & -1.897928 & -2.514010\end{array}$

$\begin{array}{lrrr}0 & 2.162319 & 6.309983 & 1.772512\end{array}$

$0-8.679318-0.677933-2.451161$

o $1.062050 \quad 7.018633 \quad 0.026105$ 
$\begin{array}{lllll}0 & -9.424871 & 0.660090 & -0.895671\end{array}$

\section{$\mathrm{C} \cdot \mathbf{1 B}$}

\begin{tabular}{|c|c|c|c|}
\hline $\mathrm{Zn}$ & 1.670848 & -1.354968 & -0.147998 \\
\hline $7 n$ & -1.222464 & -0.743619 & 1.537467 \\
\hline O & -0.284122 & -0.628786 & 3.280341 \\
\hline & -0.286580 & -1.954618 & \\
\hline & -0.177871 & -1.517783 & \\
\hline$N$ & -3.391882 & -0.34335 & \\
\hline & -2.119801 & -2.5881 & \\
\hline & 1.510026 & -2.273084 & \\
\hline $\mathrm{N}$ & 3.372019 & -2.701065 & \\
\hline & -3.936318 & 0.868011 & \\
\hline & -3.23 & & \\
\hline C & -5.30 & 1.09 & \\
\hline & -5.70 & & \\
\hline & -6.13 & & \\
\hline $\mathrm{H}$ & -7.20 & & \\
\hline & -5.56 & -1.22 & \\
\hline & -6.18 & -2.07 & \\
\hline C & -4.18 & -1.38 & \\
\hline c & -3.51 & -2.73 & \\
\hline & -3.53 & -3.2 & \\
\hline $\mathrm{H}$ & -4.10 & -3 . & \\
\hline $\mathrm{C}$ & -1.97 & -2.22 & \\
\hline & -2.81 & -1 & \\
\hline $\mathrm{H}$ & -1.9 & -3 . & \\
\hline $\mathrm{C}$ & -0.68 & $-1 \cdot 4$ & \\
\hline & -1.27 & -3 & \\
\hline $\mathrm{H}$ & -0.27 & -3.5 & \\
\hline $\mathrm{H}$ & -1.61 & -4 . & \\
\hline & $-1 \cdot 2$ & -4 . & \\
\hline C & -1.6 & -5 . & \\
\hline $\mathrm{H}$ & -2.01 & -6 . & \\
\hline & -1.47 & -5 . & -1 \\
\hline C & -1.9 & -7 & -1 \\
\hline $\mathrm{H}$ & -1.36 & -7 . & -2 \\
\hline & -1.8 & -7 . & -0 . \\
\hline $\mathrm{H}$ & -3 & -7 . & -1 \\
\hline C & -0.9 & -4 . & -1 \\
\hline $\mathrm{H}$ & -0.7 & -5 . & -2 . \\
\hline $\mathrm{C}$ & -0.5 & -3 & -1 \\
\hline C & -0.66 & -3 & -0 \\
\hline C & 0.0 & -2 . & -2 \\
\hline $\mathrm{H}$ & -0.4 & -1 . & -2 \\
\hline $\mathrm{H}$ & -0.09 & -3 & -3 \\
\hline C & 1.9 & -1 & -3 \\
\hline $\mathrm{H}$ & 1.2 & -0 . & -3 \\
\hline C & 1.73 & -1.5 & -4 \\
\hline $\mathrm{H}$ & 0.7 & -1 & -5 \\
\hline $\mathrm{H}$ & 2.005 & -0 . & -5 \\
\hline $\mathrm{H}$ & 2.383 & -2.4 & -5 \\
\hline C & 3.3 & -0.7 & \\
\hline $\mathrm{H}$ & 4.090886 & -1.4787 & -3.33887 \\
\hline & 3.5434 & 0.13 & -3 \\
\hline $\mathrm{H}$ & 3.48212 & -0.350 & -2 . \\
\hline C & 2.372765 & -3.46876 & -2.44073 \\
\hline & 1.756881 & -4.33 & -2 \\
\hline $\mathrm{H}$ & 2.76 & -3 . & -3 \\
\hline $\mathrm{C}$ & 3.525672 & -3.448059 & -1.45322 \\
\hline & & -4.23 & -1.64 \\
\hline
\end{tabular}

H $\quad 4.775554-4.825001-2.544051$

C $5.651511-4.237297-0.655107$

H $\quad 6.547565-4.837893-0.782336$

C $5.474152-3.463989 \quad 0.493680$

H $\quad 6.217915-3.445166 \quad 1.282623$

C $4.314735-2.703558 \quad 0.610013$

$\mathrm{H} \quad 4.079857 \quad-2.069474 \quad 1.462708$

O $2.248513-0.765637 \quad 1.610285$

$\mathrm{H} \quad 1.631870 \quad-0.782229 \quad 2.353085$

C $4.365463 \quad 2.439909 \quad 1.596011$

C $3.061688 \quad 2.094724 \quad 1.254648$

$\begin{array}{llll}\text { C } & 2.239563 & 3.038911 & 0.631076\end{array}$

$\begin{array}{llll}\text { C } & 2.743859 & 4.329697 & 0.374818\end{array}$

$\begin{array}{llll}\text { C } \quad 4.030921 & 4.694444 & 0.753827\end{array}$

$\begin{array}{llll}\text { C } \quad 4.833709 & 3.730780 & 1.347692\end{array}$

H $\quad 5.017024 \quad 1.718395 \quad 2.073469$

$\mathrm{H} \quad 2.689932 \quad 1.081132 \quad 1.469764$

H $\quad 4.397699 \quad 5.696619 \quad 0.577203$

C $-3.103582 \quad 4.758094-1.363046$

C $-4.223252 \quad 3.940927-1.525234$

C $-4.106688 \quad 2.581043-1.780329$

C $-2.831936 \quad 2.035178-1.873635$

C $-1.680422 \quad 2.818283-1.655476$

C $-1.836158 \quad 4.190014-1.415543$

$\mathrm{H}-3.238390 \quad 5.817008-1.179672$

$\mathrm{H}-4.983277 \quad 1.959578-1.903566$

H $-0.942834 \quad 4.786742-1.269792$

$\begin{array}{llll}0 & -0.434549 & 2.279597 & -1.684577\end{array}$

$\begin{array}{llll}0 & 0.918242 & 2.788894 & 0.387945\end{array}$

P $0.251354 \quad 1.517556-0.395023$

$\begin{array}{lrrr}0 & -0.876051 & 1.003740 & 0.469033\end{array}$

$\begin{array}{lllll}0 & 1.298981 & 0.600271 & -0.950133\end{array}$

$\mathrm{N} \quad 1.943604 \quad 5.347616 \quad-0.310175$

$\begin{array}{lrrr}\mathrm{N} & 6.208673 & 4.090430 & 1.720999\end{array}$

$\begin{array}{llll}\mathrm{N} & -5.567030 & 4.521629 & -1.399487\end{array}$

$\begin{array}{llll}\mathrm{N} & -2.741664 & 0.606561 & -2.197658\end{array}$

O $1.231988 \quad 4.979719-1.248541$

$\begin{array}{llll}0 & 2.057453 & 6.509486 & 0.068934\end{array}$

$\begin{array}{lllll}0 & -1.811322 & 0.240107 & -2.912887\end{array}$

$0-3.628553-0.125936-1.761804$

$\begin{array}{lrrr}0 & 6.578765 & 5.241448 & 1.491390\end{array}$

$\begin{array}{lllll}0 & -6.520481 & 3.741787 & -1.371067\end{array}$

$\begin{array}{llll}0 & 6.903891 & 3.216175 & 2.235761\end{array}$

$\begin{array}{llll}0 & -5.651998 & 5.744142 & -1.317127\end{array}$

\section{1-TS1}

$\begin{array}{rrrr}\text { Zn } & -0.660862 & 0.427454 & 1.448205 \\ \mathrm{Zn}-1.670078 & -0.297957 & -1.690535 \\ \mathrm{O} & -2.046840 & 1.593859 & -2.213750 \\ \mathrm{O} & -2.085333 & -0.535938 & 0.325900 \\ \mathrm{O} & -3.585315 & 2.947166 & -3.165323 \\ \mathrm{~N} & -1.782260 & -2.087506 & -2.918121 \\ \mathrm{~N} & -3.892310 & -0.482926 & -2.148957 \\ \mathrm{~N} & -0.981737 & -0.954355 & 3.218761 \\ \mathrm{~N} & -1.390551 & 1.797393 & 2.923625 \\ \mathrm{C} & -0.681375 & -2.725149 & -3.360542 \\ \mathrm{H} & 0.265153 & -2.348097 & -2.986772 \\ \mathrm{C} & -0.757262 & -3.804199 & -4.235208 \\ \mathrm{H} & 0.152826 & -4.300451 & -4.552330 \\ \mathrm{C} & -2.014984 & -4.213496 & -4.678286 \\ \mathrm{H} & -2.112415 & -5.042396 & -5.373265\end{array}$ 
$\begin{array}{llll}\text { C } & 1.982226 & 4.136581 & -3.047181\end{array}$

C $1.9512495 .148743-2.087920$

C $2.021760 \quad 4.870282-0.729158$

C $2.167889 \quad 3.548143-0.328923$

C $2.211548 \quad 2.499543-1.269905$

C $2.106485 \quad 2.817594-2.629988$

H $1.906100 \quad 4.394408-4.095949$

H $\quad 1.961601 \quad 5.665273 \quad 0.001357$

H $2.118141 \quad 2.001871-3.343769$

$\begin{array}{lllll}0 & 2.418127 & 1.211907 & -0.920278\end{array}$

O $2.471489-1.157811-1.108975$

$\begin{array}{llll}\mathrm{P} & 1.299874 & 0.020639 & -0.635479\end{array}$

$\begin{array}{llll}0 & 1.104561 & -0.425631 & 0.817277\end{array}$

O $0.354287-0.284870-1.787901$

$\mathrm{N} \quad 3.530897 \quad-3.636656-1.943279$

N $7.058356-3.133150 \quad 1.397125$

N $1.828520 \quad 6.547895 \quad-2.517714$

$\begin{array}{llll}\mathrm{N} & 2.204498 & 3.288888 & 1.111253\end{array}$

O $2.305489-3.658742-2.068082$

$\begin{aligned} & 0 \\ & 0\end{aligned} 4.309634-4.283149-2.645526$

O $2.866196 \quad 2.339315 \quad 1.524880$

$\begin{array}{llll}0 & 1.573368 & 4.063779 & 1.833242\end{array}$

$\begin{array}{llll}0 & 7.523537 & -4.190624 & 0.968600\end{array}$

$\begin{array}{llll}0 & 1.783902 & 7.410603 & -1.641327\end{array}$

$\begin{array}{llll}0 & 7.537884 & -2.484801 & 2.329637\end{array}$

$\begin{array}{lllll}0 & 1.782873 & 6.767381 & -3.726697\end{array}$

\section{C.1Int}

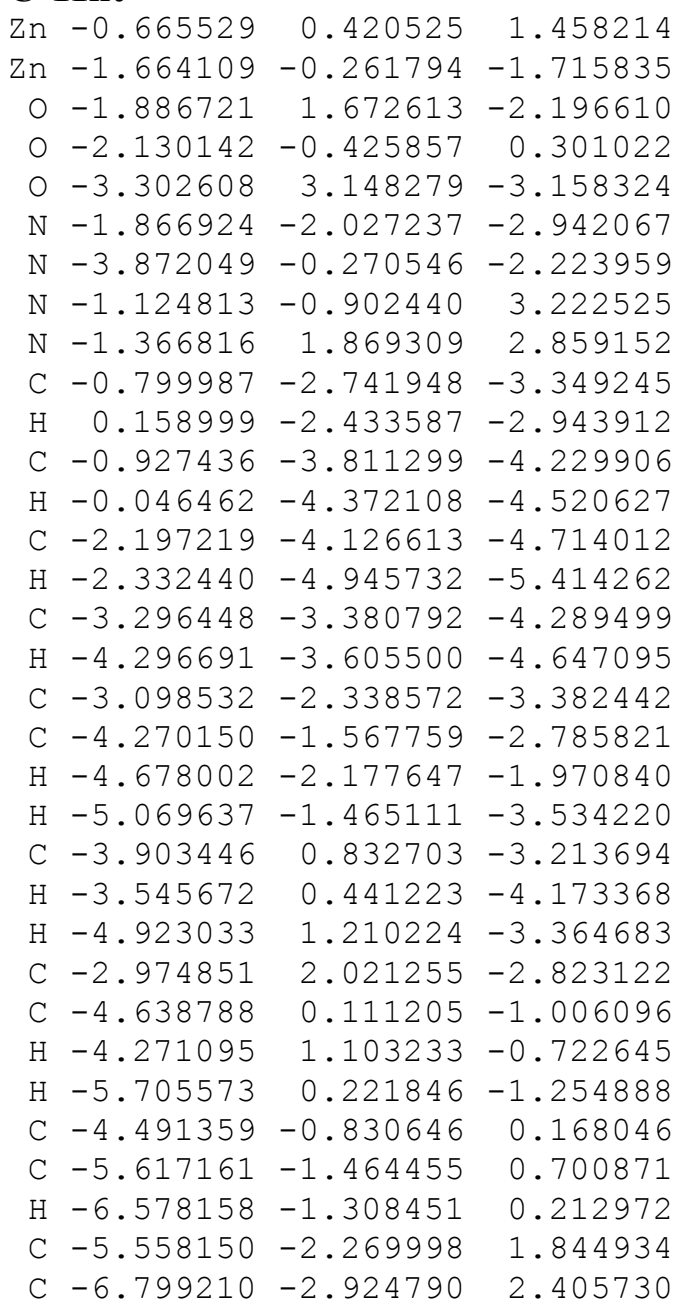


o $2.143666-3.766784-2.016746$

o $4.128506-4.464343-2.574355$

O $2.888067 \quad 2.170311 \quad 1.588706$

O $1.680512 \quad 3.966753 \quad 1.824173$

$\begin{array}{llll}0 & 7.312808 & -4.511139 & 1.061670\end{array}$

$\begin{array}{lllll}0 & 2.337597 & 7.305424 & -1.599953\end{array}$

$\begin{array}{llll}0 & 7.375233 & -2.819075 & 2.438427\end{array}$

$\begin{array}{llll}0 & 2.435066 & 6.672848 & -3.686457\end{array}$

\section{1-TS2}

Zn $\quad 1.039247-0.247164-1.578468$

$\begin{array}{llll}\text { Zn } & 1.291303 & 0.665139 & 1.758440\end{array}$

$\begin{array}{llll}\text { O } & 1.523449 & 2.600729 & 1.232887\end{array}$

o $2.128162-0.345980 \quad 0.132352$

$\begin{array}{lllll}0 & 2.735029 & 4.451008 & 1.703851\end{array}$

$\begin{array}{llll}\mathrm{N} & 1.108716 & -0.349038 & 3.592417\end{array}$

$\begin{array}{llll}\mathrm{N} & 3.304613 & 1.031812 & 2.643910\end{array}$

N $1.721333-2.176731-2.377241$

$\begin{array}{llll}\mathrm{N} & 1.891370 & 0.450759 & -3.369024\end{array}$

$\begin{array}{llll}\text { C }-0.055868 & -0.898801 & 3.991008\end{array}$

$\mathrm{H}-0.882662-0.838352 \quad 3.289010$

C $-0.179483-1.518805 \quad 5.230505$

$\mathrm{H}-1.125061-1.979069 \quad 5.491899$

C $\quad 0.927698-1.530123 \quad 6.078604$

$\mathrm{H} \quad 0.863943-1.991332 \quad 7.059871$

C $2.126359-0.948446 \quad 5.659411$

$\begin{array}{llll}\mathrm{H} & 3.001992 & -0.948936 & 6.301536\end{array}$

C $2.192110-0.376436 \quad 4.389688$

C $3.501963 \quad 0.160026 \quad 3.814510$

$\mathrm{H} \quad 4.094106 \quad-0.704650 \quad 3.493194$

$\begin{array}{llll}\mathrm{H} & 4.078598 & 0.662168 & 4.603972\end{array}$

$\begin{array}{llll}\text { C } & 3.171835 & 2.470266 & 2.982611\end{array}$

H $2.574894 \quad 2.554049 \quad 3.898393$

$\mathrm{H} \quad 4.148465 \quad 2.932414 \quad 3.174384$

$\begin{array}{llll}\text { C } & 2.440719 & 3.280916 & 1.870398\end{array}$

C $4.329050 \quad 0.856146 \quad 1.576358$

$\begin{array}{llll}\mathrm{H} & 4.082533 & 1.594425 & 0.806218\end{array}$

$\begin{array}{llll}\mathrm{H} & 5.323703 & 1.118216 & 1.967204\end{array}$

$\begin{array}{llll}\text { C } \quad 4.384773 & -0.518041 & 0.945498\end{array}$

$\begin{array}{llll}\text { C } 5.572572 & -1.254092 & 1.003098\end{array}$

$\begin{array}{llll}\mathrm{H} & 6.409948 & -0.840524 & 1.562528\end{array}$

$\begin{array}{llll}\text { C } \quad 5.733262 & -2.481612 & 0.351044\end{array}$

$\begin{array}{llll}\text { C } \quad 7.021600 & -3.263593 & 0.455318\end{array}$

H $\quad 7.144364-3.946751-0.390234$

H $\quad 7.892207 \quad-2.600589 \quad 0.479925$

H $\quad 7.050717-3.869873 \quad 1.369346$

C $4.644315-2.961079-0.380379$

H $\quad 4.733099-3.916209-0.895019$

C $3.427312-2.270613-0.460306$

$\begin{array}{llll}\text { C } \quad 3.287792 & -1.032227 & 0.209525\end{array}$

C $2.262656-2.915826-1.178709$

H $1.412469-3.016275-0.494628$

$\mathrm{H} \quad 2.566041-3.926657-1.471071$

C $0.522442-2.920314-2.944143$

$\mathrm{H}-0.147489-3.032426-2.085582$

C $0.848153-4.319022-3.492764$

$\mathrm{H} \quad 1.330877-4.968081-2.758794$

$\mathrm{H}-0.087796-4.805252-3.781630$

$\mathrm{H} \quad 1.478336-4.280443-4.387801$

C $-0.222821-2.095998-3.998869$

H $\quad 0.382460-1.922739-4.894887$ 


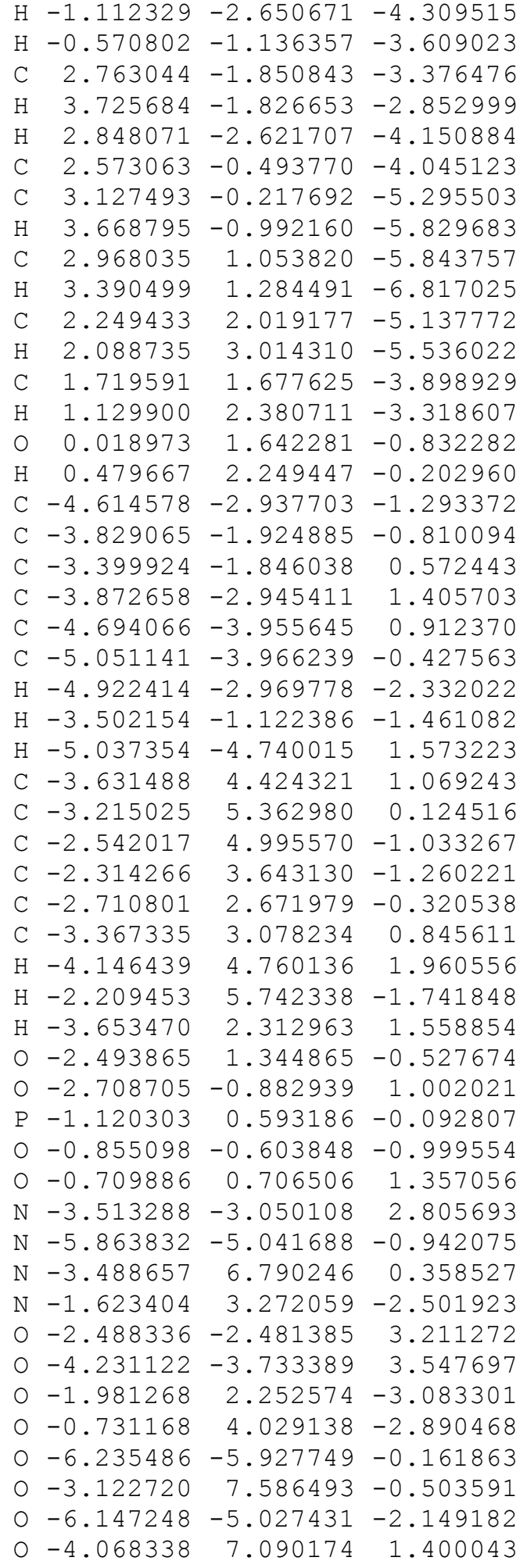

\section{$\mathbf{C} \cdot \mathbf{2} \cdot \mathbf{3}$}

$\begin{array}{rrrr}\text { Zn } & 1.496994 & 1.306745 & 0.633447 \\ \text { Zn } & 0.473720 & -1.979558 & -0.127753 \\ \mathrm{O} & 0.039093 & -2.918816 & 1.685773 \\ \mathrm{O} & 2.112547 & -0.721276 & 0.076858 \\ \mathrm{O} & -0.096256 & -4.926799 & 2.712393 \\ \mathrm{~N} & 1.674338 & -3.851250 & -0.254144 \\ \mathrm{~N} & 3.656506 & 2.070191 & 0.026378 \\ \mathrm{~N} & 2.601364 & 1.446782 & 2.540754\end{array}$

C $\quad 1.821919-4.251316-1.660194$

H $\quad 2.701042-3.739339-2.066472$

H $2.000835-5.330859-1.767596$

C $\quad 0.905800 \quad-4.832580 \quad 0.540999$

$\mathrm{H} \quad 0.101470 \quad-5.234413-0.085577$

H $\quad 1.532864 \quad-5.676896 \quad 0.856066$

C $\quad 0.233735-4.207271 \quad 1.785807$

$\begin{array}{llll}\text { C } & 2.948705 & -3.505521 & 0.438124\end{array}$

$\mathrm{H} \quad 2.659792-3.159123 \quad 1.435929$

H $3.558133-4.411949 \quad 0.570375$

$\begin{array}{llll}\text { C } \quad 3.765340 & -2.453354 & -0.269737\end{array}$

C $5.014736-2.830717-0.774568$

H $\quad 5.326061-3.866566-0.649724$

C $5.876122-1.936161-1.410426$

C $7.207427-2.375837-1.971890$

H $\quad 7.923392-1.548589-1.998018$

H $\quad 7.647937-3.180804-1.375026$

$\mathrm{H} \quad 7.109694 \quad-2.750835 \quad-2.998781$

C $5.432556-0.617965-1.514445$

$\mathrm{H} \quad 6.078613 \quad 0.116551-1.991802$

C $4.188403-0.186701-1.034737$

C $3.312345-1.108122-0.400311$

C $3.842015 \quad 1.269402-1.234611$

H $\quad 2.911393 \quad 1.372161-1.801652$

$\mathrm{H} \quad 4.639184 \quad 1.703766-1.844414$

C $\quad 3.676796 \quad 3.549798-0.281377$

H $2.844563 \quad 3.696967-0.976066$

C $4.580009 \quad 1.707888 \quad 1.116016$

H $4.966680 \quad 0.702945 \quad 0.921603$

$\mathrm{H} \quad 5.456803 \quad 2.366005 \quad 1.155242$

C $3.922202 \quad 1.677613 \quad 2.485589$

$\begin{array}{llll}\text { C } 4.683801 & 1.820991 & 3.648810\end{array}$

H $5.749834 \quad 2.013876 \quad 3.575567$

C $4.058202 \quad 1.714233 \quad 4.887954$

$\mathrm{H} \quad 4.632841 \quad 1.823232 \quad 5.803081$

C $2.685273 \quad 1.470118 \quad 4.935936$

H $2.157500 \quad 1.380018 \quad 5.878847$

C $1.992496 \quad 1.344172 \quad 3.736452$

$\mathrm{H} \quad 0.925328 \quad 1.153459 \quad 3.695673$

$\begin{array}{llll}0 & -1.547626 & -1.159181 & 2.671045\end{array}$

H $-0.996085-1.990358 \quad 2.506446$

C $-2.006649 \quad 5.273576-0.823687$

C $-0.946122 \quad 4.777062-0.115184$

C $-0.289310 \quad 3.539021-0.452426$

$\begin{array}{llll}\text { C }-0.838947 & 2.849171 & -1.602647\end{array}$

C $-1.925299 \quad 3.365193-2.326229$

C $-2.495201 \quad 4.557389-1.941460$

$\mathrm{H}-2.487540 \quad 6.204979-0.550075$

$\mathrm{H}-0.553980 \quad 5.302733 \quad 0.748762$

$\mathrm{H}-2.313595 \quad 2.820679-3.174801$

C $-5.875246-0.599100 \quad 2.803519$

C $-6.446971-1.104339 \quad 1.635214$

$\begin{array}{llll}\text { C }-5.801951 & -1.019920 & 0.409160\end{array}$

$\begin{array}{llll}\text { C }-4.563464 & -0.389883 & 0.354097\end{array}$

$\begin{array}{llll}\text { C } & -3.945811 & 0.112001 & 1.516161\end{array}$

C $-4.623010-0.003680 \quad 2.736831$

$\mathrm{H}-6.410326-0.690428 \quad 3.740312$

$\mathrm{H}-6.254636-1.424482-0.486194$

$\begin{array}{llll}\mathrm{H} & -4.130973 & 0.370549 & 3.627045\end{array}$

$\begin{array}{llll}0 & -2.726428 & 0.715875 & 1.503890\end{array}$

$\begin{array}{lllll}0 & 0.708404 & 3.163913 & 0.239849\end{array}$ 


$\begin{array}{rrrr}\mathrm{P} & -1.325332 & -0.147731 & 1.466478 \\ \mathrm{O} & -0.211671 & 0.841411 & 1.727276 \\ \mathrm{O} & -1.224159 & -0.887439 & 0.135252 \\ \mathrm{~N} & -0.296504 & 1.618021 & -2.081784 \\ \mathrm{~N} & -3.621227 & 5.086899 & -2.696438 \\ \mathrm{~N} & -7.767277 & -1.747616 & 1.698568 \\ \mathrm{~N} & -3.946153 & -0.257907 & -0.971799 \\ \mathrm{O} & 0.653191 & 1.059154 & -1.463687 \\ \mathrm{O} & -0.745418 & 1.113285 & -3.109908 \\ \mathrm{O} & -4.086824 & -1.202473 & -1.749214 \\ \mathrm{O} & -3.369466 & 0.791996 & -1.231428 \\ \mathrm{O} & -4.021688 & 4.447301 & -3.671763 \\ \mathrm{O} & -8.235392 & -2.189059 & 0.650689 \\ \mathrm{O} & -4.112247 & 6.153517 & -2.313368 \\ \mathrm{O} & -8.317201 & -1.802453 & 2.797241 \\ \mathrm{C} & 0.613895 & -3.839686 & -2.489620 \\ \mathrm{C} & 0.255519 & -4.518872 & -3.653395 \\ \mathrm{C} & -0.818457 & -4.047609 & -4.408726 \\ \mathrm{H} & 0.809462 & -5.401487 & -3.957743 \\ \mathrm{C} & -1.103311 & -2.294960 & -2.802049 \\ \mathrm{C} & -1.509677 & -2.916065 & -3.979544 \\ \mathrm{H} & -1.114613 & -4.563662 & -5.317214 \\ \mathrm{H} & -1.614956 & -1.421700 & -2.415605 \\ \mathrm{H} & -2.354682 & -2.519556 & -4.530354 \\ \mathrm{~N} & -0.065276 & -2.751483 & -2.078927 \\ \mathrm{C} & 3.397118 & 4.401949 & 0.962777 \\ \mathrm{H} & 4.230870 & 4.376597 & 1.672919 \\ \mathrm{H} & 3.272708 & 5.443635 & 0.651715 \\ \mathrm{H} & 2.479622 & 4.096992 & 1.463826 \\ \mathrm{C} & 4.970475 & 4.048370 & -0.957325 \\ \mathrm{H} & 4.886527 & 5.127419 & -1.117533 \\ \mathrm{H} & 5.854871 & 3.885124 & -0.331244 \\ \mathrm{H} & 5.157902 & 3.596889 & -1.934072 \\ & -1.96\end{array}$

\section{$\mathrm{C} \cdot 2^{+}$}

$\begin{array}{rrrr}\text { Zn } & -1.516383 & -1.403272 & 0.344754 \\ \text { Zn } & 0.218626 & 1.633685 & 0.662824 \\ \mathrm{O} & 0.321696 & 1.663531 & 2.680165 \\ \mathrm{O} & -1.417825 & 0.559377 & -0.005536 \\ \mathrm{O} & -0.393461 & 2.779579 & 4.507993 \\ \mathrm{~N} & -1.063291 & 3.424949 & 1.026055 \\ \mathrm{~N} & -2.404247 & -1.947351 & -1.557710 \\ \mathrm{~N} & -3.221561 & -2.362873 & 1.116696 \\ \mathrm{C} & -0.777367 & 4.428674 & -0.013874 \\ \mathrm{H} & -1.477448 & 4.265729 & -0.841678 \\ \mathrm{H} & -0.943979 & 5.452927 & 0.346550 \\ \mathrm{C} & -0.618843 & 3.857059 & 2.378187 \\ \mathrm{H} & 0.279834 & 4.473468 & 2.263040 \\ \mathrm{H} & -1.380821 & 4.468933 & 2.874526 \\ \mathrm{C} & -0.229998 & 2.680019 & 3.308970 \\ \mathrm{C} & -2.495621 & 3.001817 & 1.063806 \\ \mathrm{H} & -2.574681 & 2.291675 & 1.894454 \\ \mathrm{H} & -3.130594 & 3.862777 & 1.317050 \\ \mathrm{C} & -2.996769 & 2.360329 & -0.211438 \\ \mathrm{C} & -4.060344 & 2.932636 & -0.918217 \\ \mathrm{H} & -4.490299 & 3.861174 & -0.547904 \\ \mathrm{C} & -4.602184 & 2.346262 & -2.068288 \\ \mathrm{C} & -5.734624 & 3.002353 & -2.822370 \\ \mathrm{H} & -6.319393 & 2.269354 & -3.385119 \\ \mathrm{H} & -6.414997 & 3.527069 & -2.145291 \\ \mathrm{H} & -5.359681 & 3.740307 & -3.542026\end{array}$

\begin{tabular}{|c|c|c|c|}
\hline C & -4.034194 & 1.145619 & -2.505335 \\
\hline $\mathrm{H}$ & -4.423260 & 0.674196 & -3.405874 \\
\hline & -2.963405 & 0.535956 & -1.837589 \\
\hline C & -2.444117 & 1.145988 & -0.67544 \\
\hline C & -2.322751 & -0.706124 & -2.41838 \\
\hline $\mathrm{H}$ & -1.254122 & -0.533384 & -2.5841 \\
\hline $\mathrm{H}$ & -2.777947 & -0.906823 & -3.3934 \\
\hline $\mathcal{U}$ & -1.592594 & -3.075531 & -2.1821 \\
\hline $\mathrm{H}$ & -0.599551 & -2.638143 & -2.3280 \\
\hline C & -3.816520 & -2.316170 & -1.2667 \\
\hline $\mathrm{H}$ & -4.431786 & -1.423903 & -1.4265 \\
\hline $\mathrm{H}$ & -4.181671 & -3.075904 & -1.96 \\
\hline C & -4.069310 & -2.783217 & \\
\hline C & -5.177738 & -3.569887 & 0.47 \\
\hline $\mathrm{H}$ & -5.847146 & -3.90 & -0.30 \\
\hline $\mathrm{C}$ & -5.406 & -3.91 & 1.80 \\
\hline $\mathrm{H}$ & -6.262823 & -4.526118 & 2.0737 \\
\hline C & -4.517 & -3.47 & 2.75 \\
\hline $\mathrm{H}$ & -4.657 & -3.73 & 3.8 \\
\hline C & -3.430143 & -2.704915 & 2.40390 \\
\hline $\mathrm{H}$ & -2.693558 & -2.35 & 3.1191 \\
\hline O & 1.552696 & -0.61 & 3.08 \\
\hline $\mathrm{H}$ & 1.115259 & 0.284 & 3.2210 \\
\hline C & 6.0488 & -1.85 & $0.9^{\circ}$ \\
\hline $\mathrm{C}$ & 6.122815 & -1.49 & -0.36879 \\
\hline C & 4.989267 & -1.373035 & -1.15986 \\
\hline C & 3.749 & -1.63 & -0.5873 \\
\hline C & 3.637352 & -1.977493 & 0.7769 \\
\hline $\mathrm{C}$ & 4.800873 & -2.081768 & 1.545423 \\
\hline $\mathrm{H}$ & 6.959 & -1.933 & 1.5 \\
\hline $\mathrm{H}$ & 5.071236 & -1.091845 & -2.20154 \\
\hline $\mathrm{H}$ & 4.698809 & -2.337955 & 2.594064 \\
\hline O & 2.445007 & -2.234 & 1.3830 \\
\hline $\mathrm{P}$ & 1.280614 & -1.117626 & 1.613872 \\
\hline O & -0.038778 & -1.888327 & 1.517058 \\
\hline O & 1.442965 & -0.002544 & 0.58473 \\
\hline $\mathrm{N}$ & 7.438903 & -1.223621 & -0.97551 \\
\hline $\mathrm{N}$ & 2.580359 & -1.553585 & -1.469028 \\
\hline O & 2.599928 & -0.701359 & -2.3527 \\
\hline O & 1.664132 & -2.359398 & -1.29162 \\
\hline O & 7.453559 & -0.883751 & -2.156423 \\
\hline O & 8.424602 & -1.352498 & -0.256070 \\
\hline C & 0.631403 & 4.301195 & -0.577349 \\
\hline C & 1.282356 & 5.389077 & -1.16009 \\
\hline C & 2.531056 & 5.199273 & -1.749679 \\
\hline $\mathrm{H}$ & 0.813350 & 6.367738 & -1.150982 \\
\hline C & 2.406334 & 2.891430 & -1.130531 \\
\hline $\mathrm{C}$ & 3.102772 & 3.926288 & -1.743271 \\
\hline $\mathrm{H}$ & 3.051922 & 6.034951 & -2.206625 \\
\hline $\mathrm{H}$ & 2.800737 & 1.880783 & -1.09025 \\
\hline $\mathrm{H}$ & 4.070019 & 3.737505 & -2.194850 \\
\hline $\mathrm{N}$ & 1.200896 & 3.078545 & -0.558759 \\
\hline $\mathrm{C}$ & -1.441604 & -4.252740 & -1.21384 \\
\hline $\mathrm{H}$ & -2.397411 & -4.743672 & -1.00350 \\
\hline $\mathrm{H}$ & -0.783976 & -5.000772 & -1.664425 \\
\hline $\mathrm{H}$ & -0.979799 & -3.962317 & -0.26412 \\
\hline C & -2.110580 & -3.552072 & -3.54701 \\
\hline $\mathrm{H}$ & -1.397246 & -4.273880 & -3.95436 \\
\hline $\mathrm{H}$ & -3.075835 & -4.064056 & -3.47838 \\
\hline $\mathrm{H}$ & -2.19834 & -2.743918 & -4 \\
\hline
\end{tabular}




\section{1-TS1(base)}

\begin{tabular}{|c|c|c|c|}
\hline $\mathrm{n}$ & -1.583752 & 1.759295 & \\
\hline & -0.787481 & -0.955270 & -1.798608 \\
\hline O & -0.357165 & 0.199296 & \\
\hline O & -2.145427 & -0.034726 & -0.544203 \\
\hline & -1.069187 & 0.802729 & -5.421842 \\
\hline & -0.552575 & -3.120755 & -1.987 \\
\hline & -2.516791 & -1.578371 & -3.1600 \\
\hline & -2.611875 & 1.094970 & \\
\hline & -2.915012 & 3.418912 & 0.8 \\
\hline & 0.562064 & -3.762078 & $-1 \cdot 5$ \\
\hline & 1.275664 & -3.160114 & $-1 \cdot 0$ \\
\hline & 0.784159 & -5.105583 & -1.87 \\
\hline $\mathrm{H}$ & 1.695577 & -5.587553 & -1.54 \\
\hline C & -0.182110 & -5.798446 & $-2 \cdot 6$ \\
\hline & -0.0386 & -6.845180 & $-2 \cdot 8$ \\
\hline $\mathrm{C}$ & $-1 \cdot 33$ & -5.12 & $-3 \cdot 0$ \\
\hline $\mathrm{H}$ & -2.1014 & -5.639231 & -3.5 \\
\hline & -1.49 & -3.78 & -2 \\
\hline $\mathrm{C}$ & -2.76 & -3.01 & -3.0 \\
\hline $\mathrm{H}$ & -3.47 & -3.170749 & -2.21 \\
\hline & -3.21 & -3.44 & -3 \\
\hline $\mathrm{C}$ & -1.968 & -1.20 & $-4 \cdot 4$ \\
\hline $\mathrm{H}$ & -1.333397 & -2.026008 & -4.8 \\
\hline & -2.7674 & -1.060483 & -5.2 \\
\hline $\mathrm{C}$ & -1.07 & 0.06 & -4.4 \\
\hline C & 3.711539 & -0.750568 & -2.83 \\
\hline $\mathrm{H}$ & -3.4124 & 0.28 & -3 \\
\hline $\mathrm{H}$ & -4 & -0.97 & -3.5 \\
\hline C & .2025 & -0.915419 & $-1 \cdot 4$ \\
\hline C & -5.4 & -1.41 & -1 \\
\hline $\mathrm{H}$ & -6.7 & -1.70 & -2.0 \\
\hline C & -5 . & -1.5 & 0.1 \\
\hline C & 7. & -2 & 0 . \\
\hline $\mathrm{H}$ & -8 & -1.25 & 0.3 \\
\hline $\mathrm{H}$ & -7.695868 & -2.77 & -0.3 \\
\hline $\mathrm{H}$ & -7.45 & -2.5 & 1 . \\
\hline $\mathrm{C}$ & -5 . & -1.17 & 1.1 \\
\hline $\mathrm{H}$ & -5 . & -1.29 & 2.2 \\
\hline C & & -0.6 & 0 \\
\hline $\mathrm{C}$ & -3. & -0.529911 & -0.3 \\
\hline C & -2 . & -0.35 & 2.1 \\
\hline $\mathrm{H}$ & -2 . & -0.8 & \\
\hline $\mathrm{H}$ & -3.4 & -0.73 & 3.0 \\
\hline C & -1.592574 & 1.252520 & 3.4 \\
\hline $\mathrm{H}$ & -0.7 & 0.6 & \\
\hline $\mathrm{C}$ & -2.0 & 0.68320 & 4.8 \\
\hline $\mathrm{H}$ & -2.178504 & -0.398225 & 4.7 \\
\hline $\mathrm{H}$ & $-1 \cdot 2$ & 0 & 5.5 \\
\hline $\mathrm{H}$ & -2 & 1.15 & 5.1 \\
\hline C & $-1 \cdot 1$ & 2.7 & 3.5 \\
\hline $\mathrm{H}$ & -1 & & \\
\hline $\mathrm{H}$ & -0.316403 & 2.74 & 4.317 \\
\hline $\mathrm{H}$ & -0.764143 & 3.12 & 2.65 \\
\hline $\mathrm{C}$ & -3.820486 & 1.91 & 2.52 \\
\hline $\mathrm{H}$ & -4.665314 & 1.38993 & 2.0709 \\
\hline $\mathrm{H}$ & -4.071113 & 2.04 & 3.587 \\
\hline $\mathrm{C}$ & -3.746579 & 3.283998 & 1.8666 \\
\hline $\mathrm{C}$ & -4.555085 & 4.342983 & 2.2887 \\
\hline $\mathrm{H}$ & -5.216769 & & \\
\hline $\mathrm{C}$ & -4 . & 5.55 & 1.6 \\
\hline
\end{tabular}

\begin{tabular}{|c|c|c|c|}
\hline $\mathrm{H}$ & -5.110758 & 6.391716 & 1.921827 \\
\hline C & -3.621815 & 5.686045 & 0.523813 \\
\hline & -3.539560 & 6.615933 & -0.027858 \\
\hline & -2.845220 & 4.590564 & 0.162660 \\
\hline & -2.136145 & 4.605755 & -0.660377 \\
\hline & -0.911672 & 2.734977 & -1.31046 \\
\hline & -1.298203 & 2.528965 & -2.167620 \\
\hline & 3.878446 & 4.304305 & 0.18 \\
\hline & 3.175709 & 3.157491 & 0.51 \\
\hline & 3.476719 & 1.930525 & -0.0 \\
\hline & 4.52612 & 1.882250 & -1.032 \\
\hline & 5.258993 & 3.021453 & -1.35 \\
\hline & 4.91613 & 4.22 & -0.74 \\
\hline & 3.64968 & 5.26 & 0.63 \\
\hline & 2.372058 & 3.180139 & 1.24 \\
\hline & 6.074304 & 2.96771 & -2.0 \\
\hline & 3.890105 & -3.363888 & 1.1 \\
\hline & 3.653399 & -4.113954 & 2.26 \\
\hline & $2.70 s$ & -3.70 & \\
\hline & 1.974636 & -2.559467 & 2.94 \\
\hline & 2.202024 & -1.744438 & 1.798 \\
\hline $\mathrm{C}$ & 3.182850 & -2.19 & $0.8 \varepsilon$ \\
\hline $\mathrm{H}$ & 4.640769 & -3.702709 & 0.40 \\
\hline & 2.536398 & -4.286294 & 4.095 \\
\hline & 3.391431 & -1.601484 & -0.00 \\
\hline & 1.540569 & -0.606570 & 1.696 \\
\hline & 2.882191 & 0.795251 & 0.334 \\
\hline $\mathrm{P}$ & 1.281272 & 0.374670 & \\
\hline O & 0.890222 & -0.776410 & -0.664 \\
\hline O & 0.320875 & 1.333838 & 0.951 \\
\hline $\mathrm{N}$ & 4.893552 & 0.638381 & -1.72 \\
\hline $\mathrm{N}$ & 5.673083 & 5.431670 & -1.09 \\
\hline & 4.400527 & -5.346739 & 2.494 \\
\hline $\mathrm{N}$ & 0.928590 & -2.223861 & 3.9 \\
\hline O & 4.023098 & -0.219101 & -1.88354 \\
\hline O & 6.052887 & 0.539281 & -2.11605 \\
\hline O & -0.156492 & -1.827853 & 3.4 \\
\hline O & 1.180013 & -2.402334 & 5.101593 \\
\hline O & 6.579953 & 5.324041 & -1.915103 \\
\hline O & 4.175509 & -5.968127 & 3.534631 \\
\hline O & 5.351097 & 6.478585 & -0.529768 \\
\hline O & 5.207508 & -5.696055 & 1.630493 \\
\hline O & 1.255250 & 1.621808 & \\
\hline $\mathrm{H}$ & 1.228085 & 1.108683 & -2.206019 \\
\hline & 0.306685 & 2.204741 & $-1 \cdot 3$ \\
\hline
\end{tabular}

\section{DPCPMP C $\cdot 1$}

$\begin{array}{rrrr}\text { Zn } & 0.594640 & -1.806956 & -0.233252 \\ \text { Zn } & 2.389118 & 0.068366 & -1.824387 \\ \mathrm{O} & 3.603077 & -0.702640 & -3.210210 \\ \mathrm{O} & 2.280144 & -0.558933 & 0.170573 \\ \mathrm{O} & 5.622971 & -0.493175 & -4.197979 \\ \mathrm{~N} & 4.344019 & 1.063390 & -1.236964 \\ \mathrm{~N} & 0.819525 & -2.681972 & 1.834492 \\ \mathrm{~N} & 2.178062 & -3.389302 & -0.545653 \\ \mathrm{C} & 4.053130 & 2.375471 & -0.661844 \\ \mathrm{H} & 3.799592 & 2.226312 & 0.395634 \\ \mathrm{H} & 4.920810 & 3.053387 & -0.685749 \\ \mathrm{C} & 5.020458 & 1.131484 & -2.550609 \\ \mathrm{H} & 4.618655 & 1.985954 & -3.106573 \\ \mathrm{H} & 6.102733 & 1.281169 & -2.443695\end{array}$




$\begin{array}{rrrr}\mathrm{N} & -5.161796 & -2.362143 & 1.246972 \\ \mathrm{O} & -3.874774 & 3.752921 & -0.078695 \\ \mathrm{O} & -3.839279 & 5.295363 & 1.463292 \\ \mathrm{O} & -4.331567 & -2.170572 & 2.133261 \\ \mathrm{O} & -5.750833 & -3.429139 & 1.061151 \\ \mathrm{O} & 0.631879 & 7.154211 & 2.073539 \\ \mathrm{O} & -9.374356 & -0.920696 & -0.969281 \\ \mathrm{O} & 2.345819 & 5.802909 & 2.047974 \\ \mathrm{O} & -8.898677 & 0.892166 & -2.088256 \\ \mathrm{C} & 2.871012 & 3.038843 & -1.354129 \\ \mathrm{C} & 2.733595 & 4.428567 & -1.384242 \\ \mathrm{~N} & 1.938920 & 2.231541 & -1.898221 \\ \mathrm{H} & -0.594186 & -3.646328 & 3.129237 \\ \mathrm{C} & -1.334154 & -3.699686 & 1.101535 \\ \mathrm{C} & -1.724120 & -3.825070 & -1.184496 \\ \mathrm{H} & -1.431693 & -3.474336 & -2.170427 \\ \mathrm{~N} & -1.034678 & -3.315505 & -0.151721 \\ \mathrm{C} & 0.847666 & 2.769867 & -2.470271 \\ \mathrm{H} & 0.117701 & 2.071259 & -2.867451 \\ \mathrm{C} & 0.642670 & 4.144995 & -2.538757 \\ \mathrm{H} & -0.249630 & 4.536108 & -3.014697 \\ \mathrm{C} & 1.607127 & 4.987456 & -1.985375 \\ \mathrm{H} & 1.486279 & 6.066059 & -2.022931 \\ \mathrm{H} & 3.496960 & 5.056592 & -0.936401 \\ \mathrm{C} & -2.353451 & -4.619109 & 1.356873 \\ \mathrm{C} & -3.078799 & -5.140536 & 0.287026 \\ \mathrm{H} & -2.587123 & -4.900722 & 2.377948 \\ \mathrm{C} & -2.756758 & -4.742096 & -1.011220 \\ \mathrm{H} & -3.887502 & -5.841289 & 0.465926 \\ \mathrm{H} & -3.294389 & -5.126826 & -1.870815\end{array}$

\section{DPCPMP C·1Int}

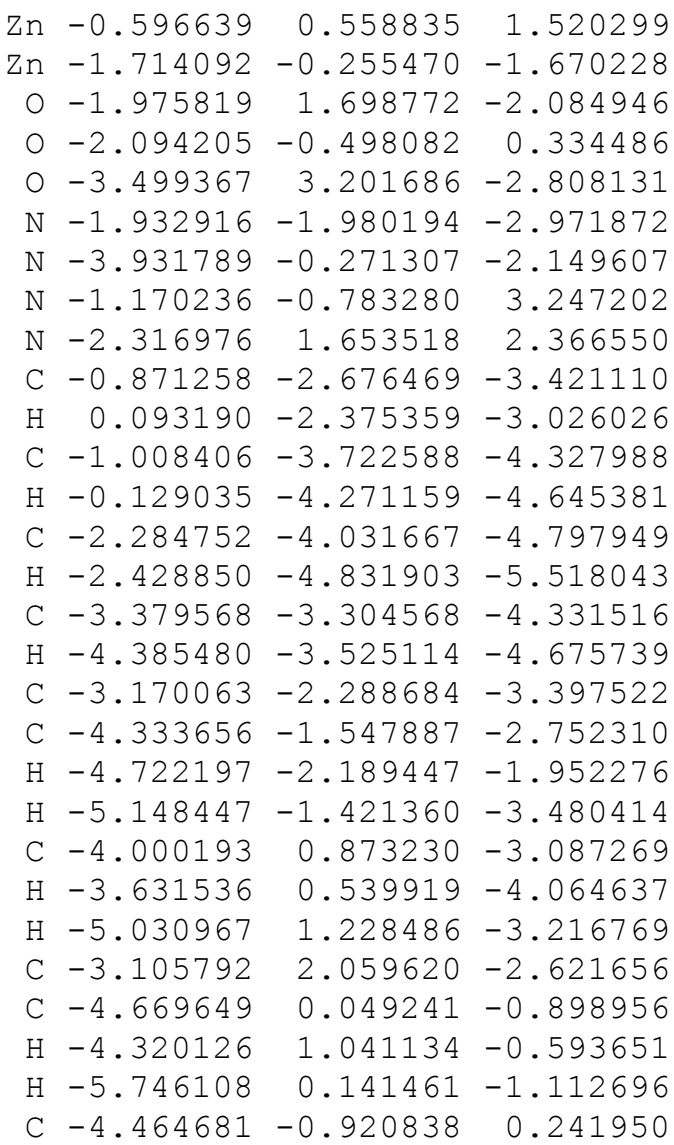




\begin{tabular}{|c|c|c|c|}
\hline C & -5.569850 & -1.574858 & 0.795253 \\
\hline $\mathrm{H}$ & -6.542779 & -1.429631 & 0.327662 \\
\hline & -5.480340 & -2.381971 & 1.935191 \\
\hline C & -6.687002 & -3.107739 & 2.482900 \\
\hline $\mathrm{H}$ & -6.576392 & -3.319134 & 3.551047 \\
\hline $\mathrm{H}$ & -7.602780 & -2.522230 & 2.350854 \\
\hline $\mathrm{H}$ & -6.844638 & -4.069815 & 1.978586 \\
\hline $\mathrm{C}$ & -4.223030 & -2.496184 & 2.5338 \\
\hline $\mathrm{H}$ & -4.117209 & -3.103664 & 3.4316 \\
\hline C & -3.086559 & -1.867465 & 2.0144 \\
\hline $\mathrm{C}$ & -3.182815 & -1.087087 & 0.834387 \\
\hline $\mathrm{C}$ & -1.749710 & -2.046142 & 2.688886 \\
\hline $\mathrm{H}$ & -1.011290 & -2.420797 & 1.971881 \\
\hline $\mathrm{H}$ & -1.847725 & -2.787823 & 3.496 \\
\hline C & 0.117077 & -1.060749 & 3.915 \\
\hline $\mathrm{C}$ & -2.102147 & -0.043882 & \\
\hline $\mathrm{H}$ & -2.797939 & -0.727131 & 4.62 \\
\hline $\mathrm{H}$ & -1.510773 & 0.441197 & 4.910 \\
\hline C & -2.892325 & 1.049642 & 3.415 \\
\hline $\mathrm{C}$ & -4.138470 & 1.460181 & 3.901 \\
\hline $\mathrm{H}$ & -4.588784 & 0.946702 & 4.7449 \\
\hline $\mathrm{C}$ & -4.790293 & 2.520834 & 3.277 \\
\hline $\mathrm{H}$ & -5.759813 & 2.853863 & 3.63 \\
\hline C & -4.188194 & 3.140450 & 2.181 \\
\hline $\mathrm{H}$ & -4.663965 & 3.963470 & 1.65 \\
\hline $\mathrm{C}$ & -2.952436 & 2.666606 & 1.752 \\
\hline $\mathrm{H}$ & -2.452749 & 3.092812 & 0.887324 \\
\hline O & 0.196322 & 1.516533 & -0.08 \\
\hline $\mathrm{H}$ & -0.358505 & 1.896033 & -0.78 \\
\hline $\mathrm{C}$ & 5.033314 & -2.048378 & 1.174666 \\
\hline $\mathrm{C}$ & 3.956438 & -1.418813 & 0.57 \\
\hline $\mathrm{C}$ & 3.275160 & -2.006073 & -0.516 \\
\hline $\mathrm{C}$ & 3.761166 & -3.264855 & -0.975965 \\
\hline $\mathrm{C}$ & 4.863803 & -3.885695 & -0.400 \\
\hline $\mathrm{C}$ & 5.481118 & -3.282038 & 0.6850 \\
\hline $\mathrm{H}$ & 5.551433 & -1.595149 & 2.010873 \\
\hline $\mathrm{H}$ & 3.634173 & -0.449157 & 0.932765 \\
\hline $\mathrm{H}$ & 5.224606 & -4.828055 & -0.789693 \\
\hline $\mathrm{C}$ & 1.973676 & 3.590240 & -3.311237 \\
\hline C & 2.509961 & 4.673792 & -2.610107 \\
\hline C & 3.009552 & 4.531416 & -1.324661 \\
\hline $\mathrm{C}$ & 3.005520 & 3.266381 & -0.745276 \\
\hline C & 2.476343 & 2.146050 & -1.424562 \\
\hline C & 1.955230 & 2.338442 & -2.716375 \\
\hline $\mathrm{H}$ & 1.580169 & 3.745835 & -4.307930 \\
\hline $\mathrm{H}$ & 3.393649 & 5.384478 & -0.782228 \\
\hline $\mathrm{H}$ & 1.525276 & 1.483700 & -3.225180 \\
\hline 0 & 2.516233 & 0.925810 & -0.867998 \\
\hline O & 2.283042 & -1.433671 & -1.160843 \\
\hline $\mathrm{P}$ & 1.225114 & -0.117729 & -0.644072 \\
\hline 0 & 0.995248 & -0.617179 & 0.782289 \\
\hline O & 0.300194 & -0.311295 & -1.845240 \\
\hline $\mathrm{N}$ & 3.127649 & -3.974269 & -2.091448 \\
\hline N & 6.615200 & -3.943712 & 1.319149 \\
\hline $\mathrm{N}$ & 2.540318 & 5.998983 & -3.238927 \\
\hline $\mathrm{N}$ & 3.511389 & 3.163566 & 0.622891 \\
\hline O & 1.909883 & -3.867351 & -2.250044 \\
\hline O & 3.854495 & -4.676023 & -2.797442 \\
\hline 0 & 4.053081 & 2.120928 & 0.981811 \\
\hline O & 3.361320 & 4.153719 & 1.348733 \\
\hline O & 6.986626 & -5.024195 & 0.85592 \\
\hline
\end{tabular}

$\begin{array}{llll}0 & 3.007722 & 6.930541 & -2.583905\end{array}$

O $7.135876-3.384148 \quad 2.287788$

$\begin{array}{lllll}0 & 2.098815 & 6.094130 & -4.383175\end{array}$

C $1.876358 \quad 0.310124 \quad 5.147005$

C $\quad 1.549162 \quad 2.266196 \quad 3.265258$

$\begin{array}{llll}\text { C } & 2.680699 & 1.448688 & 5.207343\end{array}$

$\begin{array}{llll}\mathrm{H} & 1.977815 & -0.485831 & 5.878380\end{array}$

H $1.386895 \quad 3.014645 \quad 2.496710$

C $2.521712 \quad 2.445147 \quad 4.245930$

H $3.423454 \quad 1.551782 \quad 5.992718$

H $3.138946 \quad 3.335614 \quad 4.231794$

C $0.945619 \quad 0.195894 \quad 4.113733$

$\begin{array}{llll}\mathrm{N} & 0.782722 & 1.168452 & 3.200396\end{array}$

$\mathrm{H} \quad 0.680284 \quad-1.733049 \quad 3.257668$

$\mathrm{H}-0.024178-1.579280 \quad 4.876061$

\section{DPCPMP 1-TS2}

$\begin{array}{llll}\mathrm{Zn} & 1.493450 & 1.995795 & 0.193705\end{array}$

$\begin{array}{llll}0 & 1.545747 & 2.673723 & -1.731648\end{array}$

$\begin{array}{llll}0 & 2.319829 & 0.110158 & 0.122095\end{array}$

$\begin{array}{lllll}0 & 2.685741 & 4.008443 & -3.156171\end{array}$

$\begin{array}{llll}\mathrm{N} & 1.489546 & 3.149706 & 2.016651\end{array}$

$\begin{array}{llll}\mathrm{N} & 3.513463 & 2.985836 & 0.159096\end{array}$

$\begin{array}{llll}\mathrm{N} & 2.102303 & -2.957428 & 0.005818\end{array}$

N $\quad 2.816769-1.537996-2.346869$

$\begin{array}{llll}\text { C } & 0.381017 & 3.320758 & 2.759814\end{array}$

$\begin{array}{llll}\mathrm{H} & -0.482259 & 2.738767 & 2.452063\end{array}$

C $\quad 0.358557 \quad 4.184511 \quad 3.850735$

$\mathrm{H}-0.549588 \quad 4.298158 \quad 4.431942$

C $\quad 1.518373 \quad 4.892902 \quad 4.164772$

H $\quad 1.532175 \quad 5.584121 \quad 5.002285$

$\begin{array}{llll}\text { C } & 2.663567 & 4.707465 & 3.391304\end{array}$

$\begin{array}{llll}\mathrm{H} & 3.581084 & 5.244232 & 3.611736\end{array}$

$\begin{array}{llll}\text { C } \quad 2.619108 & 3.812702 & 2.321653\end{array}$

$\begin{array}{llll}\text { C } & 3.855366 & 3.484523 & 1.496351\end{array}$

$\mathrm{H} \quad 4.400645 \quad 2.689462 \quad 2.019566$

$\mathrm{H} \quad 4.523325 \quad 4.357700 \quad 1.455899$

$\begin{array}{llll}\text { C } & 3.282159 & 4.061480 & -0.832029\end{array}$

$\mathrm{H} \quad 2.711815 \quad 4.862795 \quad-0.347631$

H $\quad 4.226806 \quad 4.488565 \quad-1.193274$

$\begin{array}{llll}\text { C } & 2.453807 & 3.553504 & -2.047010\end{array}$

$\begin{array}{llll}\text { C } \quad 4.488807 & 2.002766 & -0.382277\end{array}$

$\mathrm{H} \quad 4.135878 \quad 1.764433-1.391040$

$\begin{array}{llll}\mathrm{H} & 5.474808 & 2.480182 & -0.493268\end{array}$

$\begin{array}{llll}\text { C } & 4.639028 & 0.720029 & 0.402617\end{array}$

$\begin{array}{llll}\text { C } & 5.906002 & 0.370117 & 0.883288\end{array}$

$\begin{array}{llll}\mathrm{H} & 6.718532 & 1.085119 & 0.763441\end{array}$

$\begin{array}{llll}\text { C } & 6.174397 & -0.865002 & 1.480037\end{array}$

$\begin{array}{lll}\text { C } \quad 7.542670 & -1.197545 & 2.027156\end{array}$

$\begin{array}{llll}\mathrm{H} & 8.331935 & -0.671271 & 1.480935\end{array}$

$\mathrm{H} \quad 7.635377 \quad-0.913075 \quad 3.083155$

$\begin{array}{llll}\mathrm{H} & 7.750464 & -2.270509 & 1.964921\end{array}$

C $\quad 5.114040-1.771364 \quad 1.559038$

$\begin{array}{llll}\mathrm{H} & 5.286984 & -2.750027 & 2.004051\end{array}$

$\begin{array}{llll}\text { C } \quad 3.826666 & -1.465897 & 1.105804\end{array}$

$\begin{array}{llll}\text { C } \quad 3.557245 & -0.191778 & 0.542707\end{array}$

$\begin{array}{llll}\text { C } \quad 2.720874 & -2.475491 & 1.284324\end{array}$

H $\quad 1.907217-2.046896 \quad 1.876854$

$\mathrm{H} \quad 3.113307 \quad-3.342722 \quad 1.836633$

$\begin{array}{llll}\text { C } & 1.013075 & -3.915308 & 0.296634\end{array}$ 


\section{DPCPMP 1-TS1(base)}

$\begin{array}{llll}\mathrm{Zn} & -0.550673 & 0.367182 & -1.901299\end{array}$ $\begin{array}{llll}\mathrm{Zn} & -1.193086 & -2.137622 & 0.488206\end{array}$

$\begin{array}{llll}0 & -1.007919 & -3.478116 & -0.998255\end{array}$

$0-1.884990-0.415846-0.396041$

$\begin{array}{llll}0 & -2.103284 & -5.093077 & -2.133418\end{array}$

$\begin{array}{llll}\mathrm{N} & -3.320376 & -2.991556 & 0.466209\end{array}$

$\begin{array}{llll}\mathrm{N} & -1.443217 & 2.459233 & -1.425725\end{array}$

$\begin{array}{llll}\mathrm{N} & -2.283525 & 0.554812 & -3.317922\end{array}$

$\begin{array}{llll}\text { C }-3.887068 & -3.001027 & 1.816549\end{array}$

H $-4.391414-2.040135 \quad 1.975023$

H $-4.647269-3.787124 \quad 1.944831$

C $-3.049486-4.346381-0.062906$

$\begin{array}{llll}\mathrm{H} & -2.659117 & -4.964642 & 0.754037\end{array}$

$\mathrm{H}-3.963406-4.823162-0.440864$

C $-1.980414-4.325382-1.190797$

C $-4.132723-2.211278-0.503570$

$\mathrm{H}-3.633849-2.326909-1.471658$

$\mathrm{H}-5.134502-2.658993-0.600552$

$\begin{array}{llll}\text { C } & -4.258237 & -0.743281 & -0.176802\end{array}$

$\begin{array}{llll}\text { C }-5.517203 & -0.178853 & 0.053235\end{array}$

$\begin{array}{llll}\mathrm{H} & -6.386723 & -0.834768 & 0.055738\end{array}$

$\begin{array}{llll}\text { C }-5.700578 & 1.192681 & 0.259159\end{array}$

$\begin{array}{lll}\text { C }-7.065903 & 1.769599 & 0.552590\end{array}$

$\begin{array}{llll}\mathrm{H} & -7.147620 & 2.804533 & 0.205304\end{array}$

$\begin{array}{llll}\mathrm{H} & -7.859038 & 1.190765 & 0.068373\end{array}$

$\begin{array}{llll}\mathrm{H} & -7.280673 & 1.773897 & 1.629005\end{array}$

$\begin{array}{llll}\text { C }-4.562485 & 2.003054 & 0.200162\end{array}$

$\begin{array}{llll}\mathrm{H} & -4.669557 & 3.077344 & 0.343919\end{array}$

$\begin{array}{llll}\text { C } & -3.284670 & 1.483788 & -0.026172\end{array}$

$\begin{array}{llll}\text { C }-3.108857 & 0.087733 & -0.190935\end{array}$

$\begin{array}{llll}\text { C }-2.084228 & 2.394464 & -0.077150\end{array}$

$\begin{array}{llll}\mathrm{H} & -1.315815 & 2.030287 & 0.610483\end{array}$

$\begin{array}{llll}\mathrm{H} & -2.379337 & 3.407485 & 0.239168\end{array}$

$\begin{array}{llll}\text { C }-0.256200 & 3.335532 & -1.404521\end{array}$

$\mathrm{H} \quad 0.299084 \quad 3.105370 \quad-0.489452$

C $-2.387348 \quad 2.850167-2.491690$

$\mathrm{H}-3.180120 \quad 3.502426-2.101496$

$\mathrm{H}-1.833550 \quad 3.438133-3.233302$

$\begin{array}{llll}\text { C } & -3.009340 & 1.678997 & -3.234796\end{array}$

C $-4.242343 \quad 1.801398-3.881622$

H $-4.817847 \quad 2.716779-3.785356$

$\begin{array}{llll}\text { C } & -4.715656 & 0.732835 & -4.638825\end{array}$

H $-5.672086 \quad 0.804641-5.148340$

$\begin{array}{llll}\text { C }-3.948475 & -0.429768 & -4.731363\end{array}$

$\mathrm{H}-4.279819-1.283466-5.312317$

C $-2.737642-0.477411-4.046848$

H $-2.096240-1.354009-4.069681$

o $2.184581-1.464539-1.207836$

$\mathrm{H} \quad 2.247641-2.401907 \quad-0.988081$

$\begin{array}{llll}\text { C } & 2.307010 & 3.845786 & 2.302467\end{array}$

$\begin{array}{llll}\text { C } & 2.178622 & 2.543350 & 1.845892\end{array}$

C $1.236066 \quad 1.664056 \quad 2.426121$

$\begin{array}{llll}\text { C } & 0.427263 & 2.176858 & 3.474392\end{array}$

$\begin{array}{llll}\text { C } & 0.579917 & 3.465297 & 3.967434\end{array}$

$\begin{array}{llll}\text { C } & 1.512001 & 4.298078 & 3.361241\end{array}$

$\begin{array}{llll}\mathrm{H} & 3.029739 & 4.521087 & 1.860822\end{array}$

H $\quad 2.814778 \quad 2.179474 \quad 1.049500$

$\mathrm{H}-0.024791 \quad 3.816854 \quad 4.792874$

$\begin{array}{llll}\text { C } & 5.577523 & -2.965562 & 0.748461\end{array}$ 


\begin{tabular}{|c|c|c|c|}
\hline $\mathrm{C}$ & 6.338044 & -2.509035 & -0.327675 \\
\hline $\mathrm{C}$ & 6.009142 & -1.345931 & -1.013344 \\
\hline$C$ & 4.912123 & -0.609872 & -0.587333 \\
\hline C & 4.113472 & -1.044757 & 0.489396 \\
\hline $\mathrm{C}$ & 4.467646 & -2.231105 & 1.146228 \\
\hline $\mathrm{H}$ & 5.863890 & -3.880426 & 1.251965 \\
\hline $\mathrm{H}$ & 6.592267 & -1.021539 & -1.864583 \\
\hline$\theta$ & 3.845473 & -2.558644 & 1.971544 \\
\hline O & 3.070169 & -0.331953 & 0.966595 \\
\hline O & 1.108834 & 0.387269 & 2.105428 \\
\hline $\mathrm{P}$ & 1.456450 & -0.395089 & 0.587479 \\
\hline U & 0.943065 & 0.651501 & -0.37 \\
\hline 0 & 0.757686 & -1.697813 & 0.94 \\
\hline $\mathrm{N}$ & -0.623689 & 1.353156 & 4.08 \\
\hline$V$ & 1.657900 & 5.670101 & 3.841 \\
\hline $\mathrm{N}$ & 7.513774 & -3.277262 & -0.756 \\
\hline $\mathrm{N}$ & 4.599485 & 0.609929 & -1.33 \\
\hline O & -1.307966 & 0.647979 & 3.34 \\
\hline O & -0.781877 & 1.449607 & 5.29 \\
\hline 0 & 4.142695 & 1.571253 & -0.720681 \\
\hline O & 4.854811 & 0.607221 & -2.5 \\
\hline O & 0.946559 & 6.028357 & 4.781 \\
\hline 0 & 8.168006 & -2.837616 & -1.700455 \\
\hline 0 & 2.481730 & 6.389537 & 3.2 \\
\hline O & 7.769769 & -4.311313 & -0.1404 \\
\hline $\mathrm{H}$ & -0.532506 & 4.400979 & -1.368464 \\
\hline O & 0.214589 & -1.342964 & -2.60956 \\
\hline $\mathrm{H}$ & -0.247924 & -2.164603 & -2.37659 \\
\hline $\mathrm{H}$ & 1.280031 & -1.422135 & -1.94314 \\
\hline $\mathrm{C}$ & 0.670045 & 3.075010 & -2.5 \\
\hline C & 1.480943 & 4.074763 & -3.1192 \\
\hline C & 2.381811 & 3.739262 & -4.129770 \\
\hline $\mathrm{H}$ & 1.414885 & 5.091146 & -2.743360 \\
\hline C & 1.583520 & 1.486071 & -4.0051 \\
\hline C & 2.440827 & 2.421458 & -4.58008 \\
\hline $\mathrm{H}$ & 3.031576 & 4.497548 & -4.55635 \\
\hline $\mathrm{H}$ & 1.583891 & 0.442203 & -4.30562 \\
\hline $\mathrm{H}$ & 3.137694 & 2.117861 & -5.35296 \\
\hline $\mathrm{N}$ & 0.718641 & 1.811653 & -3.03423 \\
\hline $\mathrm{C}$ & -2.817736 & -3.134528 & 2.886932 \\
\hline C & -3.123073 & -3.605624 & 4.16552 \\
\hline $\mathrm{C}$ & -2.127091 & -3.618607 & 5.1387 \\
\hline $\mathrm{H}$ & -4.128647 & -3.948456 & 4.3886 \\
\hline $\mathrm{C}$ & -0.616265 & -2.726072 & 3.5108 \\
\hline C & -0.849288 & -3.166774 & 4.8089 \\
\hline $\mathrm{H}$ & -2.345155 & -3.976219 & 6.14059 \\
\hline $\mathrm{H}$ & 0.351049 & -2.361627 & 3.18218 \\
\hline $\mathrm{H}$ & -0.047522 & -3.154545 & 5.5 \\
\hline $\mathrm{N}$ & -1.579727 & -2.716607 & 2.57 \\
\hline
\end{tabular}

$$
\begin{aligned}
& \mathrm{C} \cdot 4 \\
& \begin{array}{llll}
0 & -0.637755 & -1.657865 & 2.205318
\end{array} \\
& \begin{array}{llll}
\text { C } & 0.178067 & -2.636729 & 2.867013
\end{array} \\
& \text { C } 1.305265-3.120134 \quad 1.943666 \\
& \text { C } 0.689846-2.109204 \quad 4.206834 \\
& \mathrm{H}-0.463997-3.508979 \quad 3.041537 \\
& \begin{array}{llll}
0 & 2.112032 & -2.008112 & 1.480032
\end{array} \\
& \mathrm{H} \quad 1.982358 \quad-3.780288 \quad 2.493367 \\
& \text { H } \quad 0.883754-3.663385 \quad 1.094999 \\
& \mathrm{H}-0.137584-1.731680 \quad 4.814206 \\
& \mathrm{H} \quad 1.184555-2.912840 \quad 4.761568
\end{aligned}
$$

$\mathrm{H} \quad 1.413870-1.301690$

P $\quad 1.796977-1.294855$

O $3.097272-1.696238-0.861633$

$0 \quad 0.616713-1.921677-0.622608$

$\begin{array}{llll}0 & 1.820357 & 0.209771 & 0.273819\end{array}$

C $4.391510-1.299116-0.701860$

$\begin{array}{llll}\text { C } \quad 4.882239 & -0.665317 & 0.450451\end{array}$

C $5.248694-1.579711-1.777973$

C $\quad 6.224349-0.305862 \quad 0.513193$

H $4.223057-0.458571 \quad 1.284073$

C $6.587390-1.222374-1.712872$

$\begin{array}{lllll}\mathrm{H} & 4.841592 & -2.078903 & -2.650497\end{array}$

C $7.062546-0.582872-0.565954$

$\begin{array}{llll}\mathrm{H} & 6.632765 & 0.184688 & 1.387948\end{array}$

H $\quad 7.268376-1.426658-2.529488$

N $8.470936-0.192818-0.496047$

o $9.189529-0.459358-1.461435$

$\begin{array}{lllll}0 & 8.861073 & 0.374093 & 0.526826\end{array}$

$\mathrm{H}-0.374867-0.677282 \quad 2.438987$

$\begin{array}{llll}\text { Zn } & 0.102819 & 1.340697 & 0.694245\end{array}$

$\begin{array}{llll}\text { Zn }-1.367254 & -1.973058 & 0.238512\end{array}$

$\begin{array}{llll}0 & -1.392463 & -4.005906 & 0.171883\end{array}$

$\begin{array}{llll}0 & -1.360465 & 0.230824 & -0.184755\end{array}$

$\begin{array}{llll}0 & -1.367666 & -5.707615 & -1.316717\end{array}$

$\begin{array}{llll}\mathrm{N}-3.520272 & -1.678865 & 0.748070\end{array}$

$\mathrm{N}-2.340784-2.269255-1.744097$

$\begin{array}{lrrr}\mathrm{N}-1.339425 & 3.193786 & 0.964124\end{array}$

$\begin{array}{llll}\mathrm{N} & 1.023017 & 2.964438 & -0.497361\end{array}$

C $-4.001177-1.210215 \quad 1.909707$

$\mathrm{H}-3.265167-1.056856 \quad 2.693980$

C $-5.351261-0.936742 \quad 2.103581$

$\mathrm{H}-5.702283-0.566009 \quad 3.060574$

C $-6.227654-1.149931 \quad 1.037678$

H $-7.288150-0.941073 \quad 1.144661$

C $-5.725571-1.632156-0.167097$

H $-6.379900-1.804478-1.015955$

C $-4.356148-1.895518-0.278358$

C $-3.776706-2.529623-1.533022$

$\mathrm{H}-4.372108-2.225004-2.404759$

$\mathrm{H}-3.906476-3.612919-1.427445$

C $-1.637674-3.500710-2.180935$

$\mathrm{H}-2.149421-3.992011-3.018187$

H $-0.635341-3.212942-2.511139$

C $-1.457947-4.522739-1.016934$

C $-2.072940-1.104956-2.629975$

$\mathrm{H}-0.984935-0.987871-2.649624$

$\mathrm{H}-2.410261-1.326066-3.654821$

$\begin{array}{llll}\text { C } & -2.727805 & 0.171751 & -2.155331\end{array}$

C $-3.731483 \quad 0.767838-2.922430$

$\begin{array}{llll}\mathrm{H} & -4.014152 & 0.294097 & -3.861531\end{array}$

C $-4.381583 \quad 1.945478-2.534432$

C $-5.443347 \quad 2.579663-3.402646$

$\mathrm{H}-6.036110 \quad 3.307349-2.839878$

$\mathrm{H}-5.005950 \quad 3.108663-4.259186$

$\mathrm{H}-6.133104 \quad 1.831800-3.808824$

C $-3.997641 \quad 2.509082-1.315829$

$\mathrm{H}-4.505259 \quad 3.407136-0.965567$

C $-2.994451 \quad 1.951958-0.511682$

$\begin{array}{llll}\text { C } & -2.325471 & 0.775267 & -0.933307\end{array}$

C $-2.694900 \quad 2.564050 \quad 0.835743$

$\mathrm{H}-2.733425 \quad 1.794627 \quad 1.614381$ 


$\begin{array}{rrrr}\mathrm{H} & -3.479836 & 3.298725 & 1.055443 \\ \mathrm{C} & -1.138193 & 3.731400 & 2.357863 \\ \mathrm{H} & -1.261218 & 2.852815 & 2.997690 \\ \mathrm{C} & -2.151028 & 4.810416 & 2.783891 \\ \mathrm{H} & -3.185461 & 4.460352 & 2.750700 \\ \mathrm{H} & -1.943737 & 5.101937 & 3.818095 \\ \mathrm{H} & -2.078613 & 5.718528 & 2.174589 \\ \mathrm{C} & 0.290692 & 4.246645 & 2.566912 \\ \mathrm{H} & 0.502207 & 5.144356 & 1.975622 \\ \mathrm{H} & 0.424580 & 4.512705 & 3.619748 \\ \mathrm{H} & 1.038486 & 3.488609 & 2.321786 \\ \mathrm{C} & -1.071057 & 4.178807 & -0.094142 \\ \mathrm{H} & -1.747432 & 3.972969 & -0.931070 \\ \mathrm{H} & -1.282115 & 5.206960 & 0.226642 \\ \mathrm{C} & 0.343803 & 4.113400 & -0.643218 \\ \mathrm{C} & 0.893033 & 5.200426 & -1.330389 \\ \mathrm{H} & 0.322544 & 6.119109 & -1.428131 \\ \mathrm{C} & 2.166734 & 5.086204 & -1.879026 \\ \mathrm{H} & 2.609958 & 5.920351 & -2.414975 \\ \mathrm{C} & 2.865462 & 3.888002 & -1.725202 \\ \mathrm{H} & 3.861821 & 3.756051 & -2.132208 \\ \mathrm{C} & 2.257371 & 2.851741 & -1.026106 \\ \mathrm{H} & 2.740913 & 1.894984 & -0.862483 \\ \mathrm{O} & -0.065117 & 0.780694 & 2.592165 \\ \mathrm{H} & 0.744306 & 0.843151 & 3.106396\end{array}$

\section{C. $4 \mathrm{~B}$}

$\begin{array}{rrrr}\text { Zn } & -1.753166 & -1.106086 & 0.209072 \\ \mathrm{Zn} & 0.294089 & 1.485424 & 0.953547 \\ \mathrm{O} & -0.017748 & 1.383664 & 2.908869 \\ \mathrm{O} & -1.332139 & 0.892681 & -0.163661 \\ \mathrm{O} & -0.715440 & 2.540337 & 4.718722 \\ \mathrm{~N} & 1.741334 & 2.967431 & 0.173392 \\ \mathrm{~N} & -0.721939 & 3.522110 & 1.259787 \\ \mathrm{~N} & -2.642701 & -1.270533 & -2.028901 \\ \mathrm{~N} & -3.776269 & -1.863869 & 0.445115 \\ \mathrm{C} & 3.018092 & 2.654959 & -0.111190 \\ \mathrm{H} & 3.265535 & 1.600671 & -0.041669 \\ \mathrm{C} & 3.957318 & 3.616485 & -0.470022 \\ \mathrm{H} & 4.977871 & 3.323213 & -0.689726 \\ \mathrm{C} & 3.552939 & 4.950387 & -0.526879 \\ \mathrm{H} & 4.259503 & 5.730742 & -0.793501 \\ \mathrm{C} & 2.230496 & 5.272044 & -0.229466 \\ \mathrm{H} & 1.885076 & 6.300759 & -0.258188 \\ \mathrm{C} & 1.341462 & 4.250843 & 0.113266 \\ \mathrm{C} & -0.128441 & 4.530596 & 0.379191 \\ \mathrm{H} & -0.658486 & 4.496885 & -0.580668 \\ \mathrm{H} & -0.246539 & 5.552478 & 0.772106 \\ \mathrm{C} & -0.413677 & 3.756810 & 2.689315 \\ \mathrm{H} & 0.592947 & 4.185164 & 2.758778 \\ \mathrm{H} & -1.111623 & 4.475248 & 3.138020 \\ \mathrm{C} & -0.409522 & 2.454580 & 3.540075 \\ \mathrm{C} & -2.186697 & 3.352151 & 1.066182 \\ \mathrm{H} & -2.495700 & 2.586305 & 1.786317 \\ \mathrm{H} & -2.710980 & 4.281859 & 1.337786 \\ \mathrm{C} & -2.591415 & 2.932153 & -0.327442 \\ \mathrm{C} & -3.451892 & 3.734901 & -1.083603 \\ \mathrm{H} & -3.777739 & 4.684800 & -0.662381 \\ \mathrm{C} & -3.921537 & 3.353213 & -2.345192 \\ \mathrm{C} & -4.824542 & 4.255629 & -3.153744 \\ \mathrm{H} & -5.472835 & 3.679737 & -3.821573\end{array}$

\begin{tabular}{|c|c|c|c|}
\hline $\mathrm{H}$ & -5.466131 & 4.863007 & -2.507622 \\
\hline & -4.249162 & 4.948579 & -3.781337 \\
\hline & -3.501088 & 2.112558 & -2.835250 \\
\hline F & -3.847447 & 1.785662 & -3.81468 \\
\hline & -2.637481 & 1.275526 & -2.11932 \\
\hline & -2.163228 & 1.687855 & -0.8 \\
\hline( & -2.166526 & -0.028068 & -2.7212 \\
\hline & -1.072908 & -0.077397 & -2.6863 \\
\hline & -2.465727 & -0.039164 & -3.7 \\
\hline & -4.111161 & -1.325180 & -1.9134 \\
\hline & -4.487250 & -0.296612 & -1.9430 \\
\hline & -4.579229 & -1.848810 & -2.757 \\
\hline 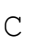 & -4.597965 & -1.939774 & -0.6114 \\
\hline 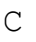 & -5.870902 & -2.503151 & -0.497 \\
\hline F & -6.519732 & -2.559412 & -1.366 \\
\hline 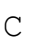 & -6.285019 & -2.990198 & \\
\hline $\mathrm{H}$ & -7.269723 & -3.435539 & 0.8501 \\
\hline C & -5.420907 & -2.905336 & 1.83 \\
\hline $\mathrm{H}$ & -5.704759 & -3.278960 & \\
\hline 0 & -4.166781 & -2.331859 & 1.64550 \\
\hline $\mathrm{H}$ & -3.427676 & -2.223653 & 2.435067 \\
\hline 0 & -1.372408 & -1.245939 & \\
\hline $\mathrm{H}$ & -1.041932 & -0.506191 & 2.64 \\
\hline C & 5.854557 & -0.984963 & 0.02 \\
\hline $\mathrm{C}$ & 6.52 & -1.248398 & -1.16 \\
\hline C & 5.850678 & -1.736795 & -2.29 \\
\hline $\mathrm{C}$ & 4.482918 & -1.953046 & -2.21218 \\
\hline C & 3.792551 & -1.684276 & -1.0 \\
\hline C & 4.482778 & -1.203502 & 0.1 \\
\hline $\mathrm{H}$ & 6.413582 & -0.615990 & 0.8752 \\
\hline $\mathrm{H}$ & 6.40 & -1.934 & -3.2 \\
\hline $\mathrm{H}$ & 3.924447 & -2.329159 & -3.062493 \\
\hline $\mathrm{H}$ & 3.955389 & -1.007554 & 1.03172 \\
\hline $\mathrm{N}$ & 7.96 & -1.004832 & -1.2 \\
\hline 0 & 8.531235 & -1.264280 & -2.31539 \\
\hline O & 8.528843 & -0.550276 & -0.254502 \\
\hline 0 & 2.452049 & -1.932642 & -1.046538 \\
\hline $\mathrm{P}$ & 1.339610 & -1.500071 & 0.102713 \\
\hline O & 0.030506 & -1.928595 & -0.51859 \\
\hline O & 1.750421 & -2.345450 & 1.38 \\
\hline O & 1.558917 & -0.037705 & 0.436781 \\
\hline C & 1.487962 & -3.769328 & 1.52866 \\
\hline $\mathrm{C}$ & 0.607639 & -4.056148 & 2.752782 \\
\hline C & 1.149709 & -3.400368 & 4.027888 \\
\hline O & -0.747424 & -3.749914 & 2.52082 \\
\hline $\mathrm{H}$ & -0.892261 & -2.757140 & 2.473718 \\
\hline $\mathrm{H}$ & 1.116224 & -2.310576 & 3.945292 \\
\hline $\mathrm{H}$ & 0.537569 & -3.700524 & 4.88229 \\
\hline $\mathrm{H}$ & 2.186756 & -3.700035 & 4.220973 \\
\hline $\mathrm{H}$ & 0.655181 & -5.149891 & 2.871904 \\
\hline $\mathrm{H}$ & 0.992600 & -4.147078 & 0.629873 \\
\hline $\mathrm{H}$ & 2.467236 & -4.248834 & 1.637670 \\
\hline $\mathrm{C}$ & -2.044827 & -2.489622 & -2.689846 \\
\hline $\mathrm{H}$ & -0.966623 & -2.319660 & -2.63159 \\
\hline C & -2.432355 & -2.674581 & -4.16959 \\
\hline $\mathrm{H}$ & -2.163678 & -1.818434 & -4.793137 \\
\hline $\mathrm{H}$ & -1.896798 & -3.542087 & -4.56685 \\
\hline $\mathrm{H}$ & -3.501987 & -2.871514 & -4.30232 \\
\hline $\mathrm{C}$ & -2.341887 & -3.774534 & -1.908215 \\
\hline $\mathrm{H}$ & -3.405859 & -4.034301 & -1.92495 \\
\hline $\mathrm{H}$ & -1.798815 & -4.602947 & -2.37311 \\
\hline
\end{tabular}


H $-2.011544-3.714712-0.869116$

\section{4-TS1}

\begin{tabular}{|c|c|c|c|}
\hline $\mathrm{Zn}$ & 2.045101 & -0.892528 & -0.262720 \\
\hline $8 n$ & -0.426150 & 1.194967 & -1.269471 \\
\hline O & 0.545880 & 1.468284 & -2.996717 \\
\hline & 1.088750 & 0.883501 & \\
\hline & 1.513443 & 2.995256 & -4.347195 \\
\hline N & -2.339989 & 2.220777 & -0.865418 \\
\hline & 0.091098 & 3.469305 & -1.11 \\
\hline & 2.462447 & -1.176309 & \\
\hline N & 4.212383 & -0.940790 & -0.09 \\
\hline & -3.516995 & 1.576438 & -0.96 \\
\hline $\mathrm{H}$ & -3.44 & 0.51 & $-1 \cdot 1$ \\
\hline C & -4.73 & 2.227 & -0.8 \\
\hline F & -5.66 & 1.667 & -0.8 \\
\hline & -4.72 & & -0 . \\
\hline $\mathrm{H}$ & -5.6 & 4.14 & -0 \\
\hline c & -3.50 & 4.26 & -0 . \\
\hline & -3.46 & & -0 . \\
\hline C & -2.32 & & -0 . \\
\hline $\mathrm{C}$ & -0.96 & 4. & -0 \\
\hline & -0.73 & & \\
\hline $\mathrm{H}$ & $-1 \cdot 0$ & 5.2 & -0 \\
\hline c & 0.13 & 3.79 & -2 . \\
\hline & -0.8 & & -2 . \\
\hline $\mathrm{H}$ & 0.6 & & -2 . \\
\hline & 0.81 & 2. & -3 \\
\hline & 1.43 & & -0 . \\
\hline $\mathrm{H}$ & 2.13 & & -1 . \\
\hline & 1.7 & & -0 . \\
\hline & 1.5 & & \\
\hline $\mathrm{C}$ & 1.8 & & \\
\hline $\mathrm{H}$ & 1.9 & 5 . & 1 . \\
\hline C & 2.0 & & \\
\hline C & 2.41 & & \\
\hline $\mathrm{H}$ & 2.85 & 3. & \\
\hline $\mathrm{H}$ & 3.1 & & \\
\hline $\mathrm{H}$ & 1.51 & & \\
\hline C & 1.96 & 2.09 & \\
\hline $\mathrm{H}$ & 2.12 & & \\
\hline $\mathrm{C}$ & 1.63 & 1.2 & \\
\hline $\mathrm{C}$ & 1.41 & 1.7 & \\
\hline C & 1.4 & -0 & \\
\hline $\mathrm{H}$ & $0.4 \varepsilon$ & -0 . & \\
\hline $\mathrm{H}$ & 1.4 & -0.4 & \\
\hline C & 3.8 & -0 & \\
\hline $\mathrm{H}$ & 3.86 & 0.28 & \\
\hline $\mathrm{H}$ & 4.29 & -1.30 & 3. \\
\hline C & 4.77 & -0.5 & \\
\hline $\mathrm{C}$ & 6.15971 & -1.05 & 1.2 \\
\hline $\mathrm{H}$ & 6.59 & -1.06 & $2.27077^{\circ}$ \\
\hline $\mathrm{C}$ & 6.9 & -1 & \\
\hline $\mathrm{H}$ & 8.041486 & -1.22314 & 0.24083 \\
\hline C & 6.3667 & -1.12627 & -1.12 \\
\hline $\mathrm{H}$ & 6.957754 & -1.1944 & -2.02747 \\
\hline C & 4.981689 & -1.026722 & -1.19764 \\
\hline $\mathrm{H}$ & 4.433154 & -1.01506 & -2.13571 \\
\hline O & 2.108567 & -0.8434 & $-2 \cdot 2$ \\
\hline $\mathrm{H}$ & 1.901521 & 0.005714 & -2.71785 \\
\hline & -5.330548 & -1.7495 & 0.2857 \\
\hline
\end{tabular}

$\begin{array}{rrrr}\mathrm{C} & -5.689816 & -1.561973 & 1.621919 \\ \mathrm{C} & -4.732674 & -1.621625 & 2.639427 \\ \mathrm{C} & -3.407229 & -1.847082 & 2.307053 \\ \mathrm{C} & -3.014231 & -2.016249 & 0.961225 \\ \mathrm{C} & -4.002282 & -1.979691 & -0.047180 \\ \mathrm{H} & -6.100863 & -1.719101 & -0.475487 \\ \mathrm{H} & -5.043284 & -1.481997 & 3.667472 \\ \mathrm{H} & -2.640989 & -1.892715 & 3.073848 \\ \mathrm{H} & -3.723184 & -2.144330 & -1.079133 \\ \mathrm{~N} & -7.079581 & -1.292058 & 1.958812 \\ \mathrm{O} & -7.374969 & -1.174448 & 3.151795 \\ \mathrm{O} & -7.890349 & -1.185648 & 1.032407 \\ \mathrm{O} & -1.707278 & -2.214749 & 0.744362 \\ \mathrm{P} & -0.851612 & -2.008580 & -0.746399 \\ \mathrm{O} & 0.539694 & -2.237070 & -0.147982 \\ \mathrm{O} & -1.593920 & -3.316082 & -1.365202 \\ \mathrm{O} & -1.340454 & -0.602650 & -1.115969 \\ \mathrm{O} & 0.067603 & -2.037742 & -2.714229 \\ \mathrm{H} & 1.113580 & -1.515091 & -2.589754 \\ \mathrm{C} & 2.164888 & -2.612917 & 2.423036 \\ \mathrm{H} & 1.120576 & -2.747129 & 2.129896 \\ \mathrm{C} & 2.306312 & -2.942856 & 3.920791 \\ \mathrm{H} & 1.693352 & -2.302959 & 4.560014 \\ \mathrm{H} & 1.974765 & -3.972437 & 4.085547 \\ \mathrm{H} & 3.343264 & -2.881309 & 4.269298 \\ \mathrm{C} & 2.995199 & -3.595523 & 1.590269 \\ \mathrm{H} & 4.067479 & -3.521942 & 1.803017 \\ \mathrm{H} & 2.684460 & -4.616131 & 1.832606 \\ \mathrm{H} & 2.834911 & -3.457026 & 0.518791 \\ \mathrm{C} & -1.343533 & -3.825693 & -2.683320 \\ \mathrm{C} & 0.043884 & -3.374719 & -3.130659 \\ \mathrm{C} & 0.261584 & -3.528952 & -4.633220 \\ \mathrm{H} & 1.255696 & -3.166150 & -4.909127 \\ \mathrm{H} & -0.478917 & -2.943531 & -5.187441 \\ \mathrm{H} & 0.184792 & -4.579714 & -4.938006 \\ \mathrm{H} & 0.803775 & -3.965565 & -2.587141 \\ \mathrm{H} & -2.105306 & -3.420975 & -3.361411 \\ \mathrm{H} & -1.448923 & -4.912617 & -2.622282\end{array}$

\section{C.4Int}

$\begin{array}{rrrr}\text { Zn } & 2.094110 & -0.793991 & -0.088021 \\ \mathrm{Zn} & -0.591263 & 0.794695 & -1.427846 \\ \mathrm{O} & 0.447456 & 0.838818 & -3.166520 \\ \mathrm{O} & 0.991151 & 0.919685 & -0.077801 \\ \mathrm{O} & 1.381469 & 2.187225 & -4.715849 \\ \mathrm{~N} & -2.574710 & 1.670194 & -1.186792 \\ \mathrm{~N} & -0.295936 & 3.102112 & -1.741996 \\ \mathrm{~N} & 2.544338 & -0.605949 & 2.183152 \\ \mathrm{~N} & 4.236751 & -0.511145 & -0.043816 \\ \mathrm{C} & -3.665387 & 0.888802 & -1.074632 \\ \mathrm{H} & -3.471828 & -0.177611 & -1.064714 \\ \mathrm{C} & -4.947187 & 1.414813 & -0.947269 \\ \mathrm{H} & -5.796087 & 0.751755 & -0.820066 \\ \mathrm{C} & -5.097944 & 2.801327 & -0.966137 \\ \mathrm{H} & -6.083038 & 3.249486 & -0.877000 \\ \mathrm{C} & -3.968274 & 3.607648 & -1.094014 \\ \mathrm{H} & -4.052246 & 4.690053 & -1.108585 \\ \mathrm{C} & -2.711020 & 3.008166 & -1.184850 \\ \mathrm{C} & -1.438646 & 3.838880 & -1.198249 \\ \mathrm{H} & -1.205155 & 4.101056 & -0.159165 \\ \mathrm{H} & -1.616349 & 4.785844 & -1.731812\end{array}$




\begin{tabular}{|c|c|c|c|}
\hline$C^{2}$ & -0.240307 & 3.136061 & -3.219699 \\
\hline $\mathrm{H}$ & -1.258304 & 3.011369 & -3.607036 \\
\hline & 0.150676 & 4.092983 & -3.590553 \\
\hline & 0.628280 & 1.981115 & -3.777885 \\
\hline & 1.003132 & 3.544200 & -1.169342 \\
\hline & 1.776776 & 2.998456 & -1.719208 \\
\hline & 1.159413 & 4.615832 & -1.371068 \\
\hline & 1.158072 & 3.273749 & 0.307954 \\
\hline & 1.353330 & 4.321814 & 1.211503 \\
\hline & 1.339640 & 5.343263 & 0.834009 \\
\hline & 1.586040 & 4.101166 & 2.574363 \\
\hline & 1.759750 & 5.252803 & 3.537182 \\
\hline & 2.308314 & 4.945838 & 4.432844 \\
\hline & 2.307110 & 6.082146 & 3.077361 \\
\hline & 0.792529 & 5.650184 & 3.870468 \\
\hline & 1.629718 & 2.773273 & \\
\hline & 1.809704 & 2.569557 & 4.0643 \\
\hline & 1.434506 & 1.688952 & 2.144432 \\
\hline & 1.185138 & 1.937246 & 0.77 \\
\hline & 1.434059 & 0.277070 & 2.68 \\
\hline & 0.508652 & -0.236513 & 2.401734 \\
\hline & 1.461828 & 0.335356 & 3.77 \\
\hline & 3.878327 & -0.002439 & 2.33 \\
\hline & 3.751718 & 1.082478 & 2.423386 \\
\hline & 4.387224 & -0.32 & 3.2 \\
\hline & 4.799131 & -0.240693 & 1.1 \\
\hline 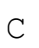 & 6.185580 & -0.118584 & 1.274986 \\
\hline & 6.619548 & 0.092338 & 2.24 \\
\hline & 6.990219 & -0.270473 & 0.14 \\
\hline$H$ & 8.069242 & -0.179747 & 0.232148 \\
\hline & 6.394204 & -0.544249 & -1.083126 \\
\hline $\mathrm{H}$ & 6.984838 & -0.669989 & -1.983599 \\
\hline$C$ & 5.010028 & -0.659927 & -1.136788 \\
\hline $\mathrm{H}$ & 4.474906 & -0.876580 & -2.056224 \\
\hline O & 2.111473 & -1.032591 & -2.220420 \\
\hline $\mathrm{H}$ & 1.696816 & -0.297212 & -2.737799 \\
\hline C & -4.998179 & -1.938747 & 0.731423 \\
\hline C & -5.300407 & -1.209775 & 1.887005 \\
\hline C & -4.296449 & -0.885209 & 2.808565 \\
\hline C & -2.987636 & -1.241719 & 2.538586 \\
\hline C & -2.638132 & -1.928455 & 1.345348 \\
\hline C & -3.688748 & -2.303358 & 0.465054 \\
\hline $\mathrm{H}$ & -5.801443 & -2.206199 & 0.054773 \\
\hline $\mathrm{H}$ & -4.561293 & -0.337137 & 3.704771 \\
\hline $\mathrm{H}$ & -2.189606 & -0.980724 & 3.226408 \\
\hline $\mathrm{H}$ & -3.452701 & -2.875234 & -0.421949 \\
\hline $\mathrm{N}$ & -6.654685 & -0.754593 & 2.110768 \\
\hline O & -6.927900 & -0.233942 & 3.198480 \\
\hline 0 & -7.474362 & -0.888279 & 1.189539 \\
\hline O & -1.358917 & -2.169582 & 1.132532 \\
\hline $\mathrm{P}$ & -0.544966 & -2.354151 & -0.514253 \\
\hline O & 0.839910 & -2.292404 & 0.187586 \\
\hline O & -1.300772 & -3.822308 & -0.552970 \\
\hline O & -1.210282 & -1.067001 & -1.066004 \\
\hline O & 0.152657 & -2.770949 & -2.140772 \\
\hline $\mathrm{H}$ & 1.457887 & -1.782849 & -2.336912 \\
\hline C & 2.422942 & -1.983816 & 2.79497 \\
\hline $\mathrm{H}$ & 1.410446 & -2.300378 & 2.530490 \\
\hline $\mathrm{C}$ & 2.575544 & -2.018075 & 4.326219 \\
\hline $\mathrm{H}$ & 1.863767 & -1.369400 & 4.84207 \\
\hline & 2.38 & -3.038366 & 4.67321 \\
\hline
\end{tabular}

$\begin{array}{rrrr}\mathrm{H} & 3.585605 & -1.749380 & 4.656081 \\ \mathrm{C} & 3.390749 & -2.980183 & 2.147969 \\ \mathrm{H} & 4.440440 & -2.730306 & 2.339783 \\ \mathrm{H} & 3.210653 & -3.973108 & 2.570173 \\ \mathrm{H} & 3.231960 & -3.058511 & 1.070226 \\ \mathrm{C} & -1.081446 & -4.650962 & -1.691933 \\ \mathrm{C} & 0.221506 & -4.169007 & -2.329355 \\ \mathrm{C} & 0.363823 & -4.524683 & -3.800607 \\ \mathrm{H} & 1.299773 & -4.129798 & -4.206627 \\ \mathrm{H} & -0.464133 & -4.102049 & -4.378599 \\ \mathrm{H} & 0.368077 & -5.612869 & -3.932566 \\ \mathrm{H} & 1.071750 & -4.585245 & -1.759403 \\ \mathrm{H} & -1.919353 & -4.532649 & -2.392644 \\ \mathrm{H} & -1.036977 & -5.689313 & -1.349426\end{array}$

\section{4-TS2}

$\begin{array}{lrrr}\text { Zn } \quad 2.178773 & 0.627688 & 0.022769\end{array}$

Zn $-0.685503-0.450057 \quad 1.520688$

$\begin{array}{lllll}0 & 0.352368 & -0.319710 & 3.262720\end{array}$

$\begin{array}{llll}0 & 0.894265 & -0.912010 & 0.264268\end{array}$

O $1.222218-1.543220 \quad 4.947162$

$\begin{array}{llll}\mathrm{N} & -2.742743 & -1.077523 & 1.398224\end{array}$

$\begin{array}{llll}\mathrm{N} & -0.634005 & -2.680959 & 2.172221\end{array}$

$\begin{array}{llll}\mathrm{N} & 2.548531 & 0.145093 & -2.181418\end{array}$

$\begin{array}{llll}\mathrm{N} & 4.260452 & 0.092412 & 0.036494\end{array}$

C $-3.736229-0.202333 \quad 1.150873$

$\begin{array}{llll}\mathrm{H} & -3.425843 & 0.819540 & 0.965014\end{array}$

C $-5.071210-0.590511 \quad 1.092949$

$\begin{array}{llll}\mathrm{H} & -5.838725 & 0.131083 & 0.835202\end{array}$

C $-5.381267-1.928845 \quad 1.331650$

$\mathrm{H}-6.412132-2.267981 \quad 1.297759$

C $-4.352301-2.830586 \quad 1.599229$

$\mathrm{H}-4.561269-3.880376 \quad 1.781031$

C $-3.033855-2.375973 \quad 1.604210$

C $-1.868885-3.342398 \quad 1.741552$

$\mathrm{H}-1.686182-3.775957 \quad 0.750954$

$\mathrm{H}-2.148300-4.174522 \quad 2.406223$

C $-0.551505-2.507811 \quad 3.639803$

$\mathrm{H}-1.541587-2.216460 \quad 4.009334$

$\mathrm{H}-0.258264-3.438207 \quad 4.143422$

C $0.449732-1.387516 \quad 4.015782$

C $0.591173-3.356414 \quad 1.663750$

H $\quad 1.436127-2.851381 \quad 2.143210$

$\mathrm{H} \quad 0.609665-4.404903 \quad 2.000070$

C $0.757962-3.294842 \quad 0.164333$

C $0.804095-4.461729-0.603924$

$\mathrm{H} \quad 0.669810-5.420172-0.104749$

C $1.035639-4.437109-1.984210$

$\begin{array}{lllll}\text { C } & 1.046341 & -5.708537 & -2.800807\end{array}$

H $\quad 1.638388-5.592342-3.713655$

H $\quad 1.465037-6.546454-2.234140$

H $\quad 0.033444 \quad-5.999315-3.107099$

C $1.232365-3.187310-2.580404$

H $1.411402-3.137271-3.653231$

C $1.189411-1.989458-1.854972$

C $0.942586-2.041790-0.465528$

C $\quad 1.336484-0.663670-2.566805$

H $\quad 0.479244 \quad-0.013568-2.352407$

H $\quad 1.347567-0.862074-3.644795$

C $3.808966-0.609939-2.279261$

H $\quad 3.568753-1.679472-2.275915$ 


\begin{tabular}{|c|c|c|c|}
\hline $\mathrm{H}$ & 4.338403 & -0.417453 & -3.220418 \\
\hline $\mathrm{C}$ & 4.766574 & -0.369298 & -1.120129 \\
\hline & 6.125045 & -0.678851 & -1.235046 \\
\hline & 6.515777 & -1.043068 & -2.180173 \\
\hline & 6.957560 & -0.514936 & -0.131664 \\
\hline & 8.015091 & -0.750646 & -0.205061 \\
\hline & 6.418155 & -0.041725 & 1.066174 \\
\hline & 7.032264 & 0.099682 & 1.948401 \\
\hline & 5.061140 & 0.253801 & 1.107977 \\
\hline $\mathrm{H}$ & 4.571590 & 0.629810 & 2.000951 \\
\hline & 2.230090 & 1.167515 & 2.150700 \\
\hline & 1.666396 & 0.587808 & 2.736571 \\
\hline $\mathrm{C}$ & -4.654960 & 1.972313 & -1.411206 \\
\hline $\mathrm{C}$ & -4.986687 & 0.816609 & -2.139254 \\
\hline C & -3.978813 & 0.072227 & -2.776667 \\
\hline $\mathrm{C}$ & -2.657271 & 0.439535 & -2.628514 \\
\hline$C$ & -2.264571 & 1.566839 & -1.8275 \\
\hline$C$ & -3.335137 & 2.347017 & $-1.267 \varepsilon$ \\
\hline $\mathrm{H}$ & -5.45510 & 2.552770 & -0.965 \\
\hline $\mathrm{H}$ & -4.262787 & -0.800572 & -3.3535 \\
\hline $\mathrm{H}$ & -1.872021 & -0.144339 & -3.10004 \\
\hline $\mathrm{H}$ & -3.074910 & 3.241619 & -0.71625 \\
\hline N & -6.345389 & 0.365404 & -2.1818 \\
\hline O & -6.640970 & -0.573007 & -2.93804 \\
\hline O & -7.174963 & 0.918707 & -1.433 \\
\hline O & -1.019570 & 1.828209 & -1.62858 \\
\hline $\mathrm{P}$ & -0.157666 & 2.583831 & 0.347991 \\
\hline O & 1.173354 & 2.288890 & -0.35699 \\
\hline O & -0.897129 & 3.946262 & -0.156302 \\
\hline O & -0.978618 & 1.466778 & 0.98576 \\
\hline O & 0.416578 & 3.340926 & 1.777 \\
\hline $\mathrm{H}$ & 1.808211 & 2.047052 & 2.217406 \\
\hline C & 2.554953 & 1.449639 & -2.95191 \\
\hline $\mathrm{H}$ & 1.590731 & 1.903866 & -2.707 \\
\hline C & 2.653795 & 1.284460 & -4.478505 \\
\hline $\mathrm{H}$ & 1.836727 & 0.692347 & -4.896249 \\
\hline $\mathrm{H}$ & 2.597496 & 2.274241 & -4.94111 \\
\hline $\mathrm{H}$ & 3.602582 & 0.835717 & -4.795921 \\
\hline C & 3.653534 & 2.394069 & -2.45253 \\
\hline $\mathrm{H}$ & 4.660533 & 2.012336 & -2.657 \\
\hline $\mathrm{H}$ & 3.558933 & 3.352361 & -2.971395 \\
\hline $\mathrm{H}$ & 3.560562 & 2.600048 & -1.38380 \\
\hline $\mathrm{C}$ & -0.793063 & 5.038728 & 0.77335 \\
\hline C & 0.445201 & 4.757933 & 1.629560 \\
\hline C & 0.437325 & 5.437856 & 2.98774 \\
\hline $\mathrm{H}$ & 1.331535 & 5.170521 & 3.557858 \\
\hline $\mathrm{H}$ & -0.441584 & 5.132775 & 3.563784 \\
\hline $\mathrm{H}$ & 0.422838 & 6.527236 & 2.86998 \\
\hline $\mathrm{H}$ & 1.347940 & 5.047663 & 1.06704 \\
\hline $\mathrm{H}$ & -1.698155 & 5.068724 & 1.39351 \\
\hline $\mathrm{H}$ & -0.715912 & 5.960538 & 0.19130 \\
\hline
\end{tabular}

\section{C.5.6}

$\begin{array}{rrrr}\text { Zn } & -0.973777 & 0.257623 & 0.388658 \\ \text { Zn } & 2.464532 & 0.501625 & -0.757880 \\ \mathrm{O} & 1.799353 & 0.895478 & -2.625031 \\ \mathrm{O} & 1.032875 & -0.635231 & 0.133209 \\ \mathrm{O} & 1.742526 & 0.113549 & -4.741883 \\ \mathrm{~N} & 4.617643 & 0.459395 & -0.303115 \\ \mathrm{~N} & 3.257429 & -1.342902 & -1.872845 \\ \mathrm{~N} & -1.047480 & -0.840825 & 2.538441\end{array}$

N $-1.813504-1.747234-0.023128$

C $5.250639 \quad 1.496976 \quad 0.273490$

$\begin{array}{llll}\mathrm{H} & 4.606588 & 2.308791 & 0.596806\end{array}$

C $6.631848 \quad 1.524335 \quad 0.436449$

$\begin{array}{llll}\mathrm{H} & 7.106531 & 2.378483 & 0.906410\end{array}$

C $7.3774140 .439606-0.026416$

$\begin{array}{llll}\mathrm{H} & 8.458699 & 0.429934 & 0.073636\end{array}$

C $6.718801-0.631476-0.626147$

H $7.269832-1.488251-1.001198$

C $5.327662-0.595016-0.743290$

C $4.543857-1.765031-1.313170$

$\mathrm{H} \quad 4.338605-2.461980-0.491554$

H $5.161436-2.306727-2.045930$

C $3.375845-0.809989-3.247847$

H $\quad 4.297943-0.220429-3.313203$

H $\quad 3.436171-1.615188-3.991459$

$\begin{array}{llll}\text { C } & 2.195272 & 0.125853 & -3.610487\end{array}$

C $2.212410-2.404102-1.810191$

$\mathrm{H} \quad 1.329963-1.984234-2.304484$

$\mathrm{H} \quad 2.530504 \quad-3.277054-2.401094$

C $1.856043-2.831306-0.408373$

$\begin{array}{llll}\text { C } & 2.061119 & -4.154191 & 0.002348\end{array}$

$\mathrm{H} \quad 2.513634-4.852201-0.700692$

$\begin{array}{llll}\text { C } & 1.692517 & -4.609808 & 1.271389\end{array}$

$\begin{array}{llll}\text { C } & 1.894501 & -6.049544 & 1.683919\end{array}$

$\mathrm{H} \quad 2.203246 \quad-6.128253 \quad 2.731698$

H $\quad 0.972584-6.635355 \quad 1.575584$

$\mathrm{H} \quad 2.660307 \quad-6.537171 \quad 1.073071$

C $\quad 1.109137-3.679664 \quad 2.140360$

H $\quad 0.831419-3.996956 \quad 3.144662$

$\begin{array}{llll}\text { C } & 0.882737 & -2.350707 & 1.773623\end{array}$

$\begin{array}{llll}\text { C } & 1.258551 & -1.907334 & 0.483868\end{array}$

$\begin{array}{llll}\text { C } & 0.339530 & -1.359143 & 2.774040\end{array}$

$\begin{array}{llll}\mathrm{H} & 0.983573 & -0.473838 & 2.789384\end{array}$

H $\quad 0.389855-1.830221 \quad 3.763849$

$\begin{array}{llll}\text { C }-1.389800 & 0.132008 & 3.647907\end{array}$

$\begin{array}{llll}\mathrm{H}-0.566096 & 0.851511 & 3.618980\end{array}$

C $-1.451713-0.497577 \quad 5.053445$

$\mathrm{H}-0.518003-0.971908 \quad 5.365578$

$\mathrm{H}-1.670383 \quad 0.288763 \quad 5.782241$

$\mathrm{H}-2.255500-1.238351 \quad 5.130060$

$\begin{array}{llll}\text { C }-2.678509 & 0.910741 & 3.373211\end{array}$

$\begin{array}{llll}\mathrm{H} & -3.574840 & 0.293633 & 3.507862\end{array}$

$\mathrm{H}-2.750055 \quad 1.729984 \quad 4.096437$

H $-2.694831 \quad 1.332206 \quad 2.366749$

C $-2.027304-1.933931 \quad 2.418652$

$\mathrm{H}-1.816811-2.754542 \quad 3.120463$

H $-3.011425-1.538125 \quad 2.688776$

C $-2.156735-2.516015 \quad 1.019655$

C $-2.685348-3.797600 \quad 0.833345$

H $-2.949691-4.402456 \quad 1.695270$

C $-2.861991-4.279219-0.459841$

$\mathrm{H}-3.275160-5.270015-0.624178$

C $-2.495358-3.474458-1.539608$

H $-2.613632-3.810189-2.563889$

C $-1.970994-2.215115-1.273114$

H $-1.661522-1.543289-2.067646$

$\begin{array}{lllll}0 & -0.686874 & 0.865575 & -1.726838\end{array}$

$\begin{array}{llll}\text { C } & -5.428546 & 0.330346 & -2.133080\end{array}$

$\begin{array}{llll}\text { C } & -6.540526 & 0.677121 & -1.355535\end{array}$

C $-6.370237 \quad 1.221166-0.074457$ 


$\begin{array}{rrrr}\mathrm{C} & -5.096341 & 1.403522 & 0.428948 \\ \mathrm{C} & -3.933562 & 1.047046 & -0.321580 \\ \mathrm{C} & -4.153105 & 0.511167 & -1.627754 \\ \mathrm{H} & -5.590942 & -0.072424 & -3.125959 \\ \mathrm{H} & -7.249185 & 1.491153 & 0.498950 \\ \mathrm{H} & -4.952873 & 1.838110 & 1.413138 \\ \mathrm{H} & -3.289807 & 0.257718 & -2.234554 \\ \mathrm{~N} & -7.871828 & 0.475322 & -1.878169 \\ \mathrm{O} & -8.835497 & 0.796951 & -1.169080 \\ \mathrm{O} & -7.990529 & -0.013854 & -3.010312 \\ \mathrm{O} & -2.744614 & 1.237608 & 0.171478 \\ \mathrm{P} & 1.013379 & 2.864186 & 0.853249 \\ \mathrm{O} & -0.047344 & 1.888150 & 1.294410 \\ \mathrm{O} & 1.476076 & 3.851802 & 2.068700 \\ \mathrm{O} & 2.262604 & 2.264307 & 0.224754 \\ \mathrm{C} & 0.765819 & 5.098327 & 1.981916 \\ \mathrm{C} & 0.535143 & 5.348721 & 0.474252 \\ \mathrm{O} & 0.369426 & 4.028524 & -0.088254 \\ \mathrm{H} & -1.243663 & 1.642801 & -1.850741 \\ \mathrm{H} & -0.184894 & 5.015763 & 2.521719 \\ \mathrm{H} & 1.383220 & 5.870816 & 2.445976 \\ \mathrm{H} & 0.158385 & 0.981743 & -2.232375 \\ \mathrm{C} & 1.679913 & 6.080315 & -0.216542 \\ \mathrm{H} & 2.633062 & 5.575186 & -0.029644 \\ \mathrm{H} & 1.510323 & 6.109273 & -1.296133 \\ \mathrm{H} & 1.747404 & 7.109689 & 0.153018 \\ \mathrm{H} & -0.409298 & 5.878060 & 0.314544\end{array}$

\section{$\mathbf{C} \cdot \mathbf{5}^{+}$}

$\begin{array}{rrrr}\text { Zn } & 1.537553 & 0.611353 & 0.216534 \\ \text { Zn } & -1.842421 & -0.002248 & 0.317608 \\ \mathrm{O} & -1.657253 & 0.490819 & 2.268195 \\ \mathrm{O} & 0.047004 & -0.727793 & -0.043346 \\ \mathrm{O} & -1.692014 & -0.428564 & 4.327839 \\ \mathrm{~N} & -3.734761 & -0.458865 & -0.601923 \\ \mathrm{~N} & -2.381678 & -1.999869 & 1.250933 \\ \mathrm{~N} & 2.790592 & -0.281452 & -1.457772 \\ \mathrm{~N} & 3.262202 & -0.032404 & 1.302974 \\ \mathrm{C} & -4.437754 & 0.439128 & -1.318299 \\ \mathrm{H} & -3.934303 & 1.381572 & -1.508731 \\ \mathrm{C} & -5.722585 & 0.176287 & -1.778648 \\ \mathrm{H} & -6.257505 & 0.923959 & -2.353250 \\ \mathrm{C} & -6.298719 & -1.057758 & -1.473745 \\ \mathrm{H} & -7.304351 & -1.294503 & -1.807206 \\ \mathrm{C} & -5.570055 & -1.983632 & -0.729680 \\ \mathrm{H} & -5.992285 & -2.950014 & -0.473372 \\ \mathrm{C} & -4.279133 & -1.656795 & -0.312027 \\ \mathrm{C} & -3.406276 & -2.657091 & 0.428700 \\ \mathrm{H} & -2.889291 & -3.269717 & -0.320229 \\ \mathrm{H} & -4.036889 & -3.338550 & 1.018058 \\ \mathrm{C} & -2.898473 & -1.543642 & 2.566139 \\ \mathrm{H} & -3.905812 & -1.136824 & 2.421973 \\ \mathrm{H} & -2.961339 & -2.368804 & 3.285552 \\ \mathrm{C} & -2.006548 & -0.423571 & 3.153710 \\ \mathrm{C} & -1.167422 & -2.844416 & 1.445258 \\ \mathrm{H} & -0.552929 & -2.322118 & 2.186046 \\ \mathrm{H} & -1.452918 & -3.808100 & 1.891810 \\ \mathrm{C} & -0.348879 & -3.080818 & 0.197141 \\ \mathrm{C} & -0.114887 & -4.380507 & -0.262805 \\ \mathrm{H} & -0.593226 & -5.210092 & 0.254662 \\ \mathrm{C} & 0.732631 & -4.650984 & -1.344054\end{array}$
C $\quad 0.943967-6.062097-1.839986$

$\mathrm{H} \quad 1.892339-6.160204-2.375718$

H $\quad 0.948438-6.780730-1.014838$

$\mathrm{H} \quad 0.147824-6.366662-2.530416$

C $\quad 1.362907-3.561715-1.953222$

H $\quad 2.032182-3.742055-2.792447$

C $\quad 1.154277-2.240458-1.534177$

C $0.277457-1.995055-0.457112$

C $\quad 1.821078-1.099997-2.271327$

$\mathrm{H} \quad 1.068775-0.389645-2.631294$

$\mathrm{H} \quad 2.327507-1.514045-3.149781$

C $\quad 3.345511 \quad 0.855884 \quad-2.293540$

H $\quad 2.457029 \quad 1.407951-2.613187$

C $4.121409 \quad 0.414133-3.545950$

$\mathrm{H} \quad 3.522632-0.194376-4.227024$

$\mathrm{H} \quad 4.427298 \quad 1.306150-4.100072$

H $\quad 5.035040-0.139085-3.303550$

C $4.198479 \quad 1.808943-1.449309$

$\mathrm{H} \quad 5.121214 \quad 1.338804 \quad-1.093625$

$\mathrm{H} \quad 4.487736 \quad 2.666046-2.063601$

$\mathrm{H} \quad 3.651872 \quad 2.202633-0.588082$

C $3.838755-1.114169-0.831434$

H $3.475432-2.147216-0.797626$

$\mathrm{H} \quad 4.758888-1.131170-1.426135$

$\begin{array}{llll}\text { C } \quad 4.178393 & -0.720890 & 0.597927\end{array}$

C $5.379184-1.130397 \quad 1.182521$

H $6.107189-1.677604 \quad 0.591912$

C $5.622393-0.832852 \quad 2.520681$

H $6.550535-1.143840 \quad 2.990217$

$\begin{array}{llll}\text { C } \quad 4.659282 & -0.130981 & 3.248134\end{array}$

H $4.807087 \quad 0.116919 \quad 4.293062$

$\begin{array}{llll}\text { C } & 3.492095 & 0.254196 & 2.601522\end{array}$

$\mathrm{H} \quad 2.703427 \quad 0.801566 \quad 3.106583$

$\begin{array}{llll}\text { O } & 0.688239 & 1.510543 & 2.014070\end{array}$

$\mathrm{P}-0.501322 \quad 2.798559-0.763277$

$\begin{array}{llll}0 & 0.898627 & 2.207308 & -0.862558\end{array}$

$\begin{array}{llll}0 & -0.739113 & 3.832977 & -1.983999\end{array}$

$0-1.632845 \quad 1.797119-0.631457$

$\begin{array}{llll}0 & -0.584403 & 3.891617 & 0.446556\end{array}$

$\mathrm{H} \quad 0.565734 \quad 2.469054 \quad 1.994050$

$\mathrm{H}-0.207219 \quad 1.133616 \quad 2.301618$

C $-1.158726 \quad 5.121324-1.482409$

C $-0.541609 \quad 5.250085-0.080441$

C $-1.277014 \quad 6.188092 \quad 0.854703$

$\mathrm{H}-0.799703 \quad 6.205768 \quad 1.837551$

$\mathrm{H}-2.318165 \quad 5.876450 \quad 0.978026$

$\mathrm{H}-1.258798 \quad 7.206056 \quad 0.451344$

$\mathrm{H} \quad 0.514753 \quad 5.535911-0.167273$

$\mathrm{H}-2.253813 \quad 5.151351-1.437780$

$\mathrm{H}-0.797380 \quad 5.884436-2.173834$

\section{4-TS1(base)}

Zn 2.161094 $-0.616905-0.112479$

$\mathrm{Zn}-0.513467 \quad 0.990240-1.519687$

○ $0.609491 \quad 1.231470-3.161339$

$\begin{array}{llll}0 & 0.870099 & 0.979076 & 0.031335\end{array}$

$\begin{array}{llll}\mathrm{O} & 1.538281 & 2.748998 & -4.550038\end{array}$

$\mathrm{N}-2.564748 \quad 1.792704-1.511975$

$\mathrm{N}-0.281525 \quad 3.303902-1.541072$

$\begin{array}{llll}\mathrm{N} & 2.289215 & -0.731778 & 2.250331\end{array}$

$\begin{array}{llll}\mathrm{N} & 4.295901 & -0.435925 & 0.326778\end{array}$ 


\begin{tabular}{|c|c|c|c|}
\hline $\mathrm{C}$ & -3.631205 & 1.013390 & -1.762705 \\
\hline 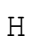 & -3.412397 & -0.041326 & -1.886606 \\
\hline C & -4.921731 & 1.525544 & -1.849310 \\
\hline $\mathrm{H}$ & -5.757094 & 0.864223 & -2.049968 \\
\hline $\mathrm{C}$ & -5.104765 & 2.897141 & -1.674854 \\
\hline $\mathrm{H}$ & -6.096749 & 3.334000 & -1.7393 \\
\hline C & -3.996819 & 3.703622 & -1.42251 \\
\hline $\mathrm{H}$ & -4.103820 & 4.775643 & -1.2897 \\
\hline C & -2.732855 & 3.116676 & -1.3412 \\
\hline $\mathrm{C}$ & -1.493761 & 3.930628 & -1.0110 \\
\hline $\mathrm{H}$ & -1.396474 & 3.980498 & \\
\hline $\mathrm{H}$ & -1.622504 & 4.964759 & -1.36813 \\
\hline $\mathrm{C}$ & -0.110360 & 3.522790 & -2.9946 \\
\hline $\mathrm{H}$ & -1.094995 & 3.471359 & -3.4 \\
\hline $\mathrm{H}$ & 0.315951 & 4.511660 & -3.2 \\
\hline $\mathrm{C}$ & 0.782998 & 2.434546 & -3.6 \\
\hline $\mathrm{C}$ & 0.951941 & 3.706451 & -0.8 \\
\hline $\mathrm{H}$ & 1.781508 & 3.23042 & $-1 \cdot 3$ \\
\hline $\mathrm{H}$ & 1.097339 & 4.795177 & -0.89 \\
\hline C & 0.991823 & 3.295927 & 0.6 \\
\hline C & 1.113230 & 4.2572 & \\
\hline $\mathrm{H}$ & 1.116126 & 5.308909 & 1.36 \\
\hline C & 1.252955 & 3.9137 & 2. \\
\hline $\mathrm{C}$ & 1.347004 & 4.972231 & \\
\hline $\mathrm{H}$ & 1.889724 & 4.605727 & 4.94 \\
\hline $\mathrm{H}$ & 1.862851 & 5.866961 & 3.7 \\
\hline $\mathrm{H}$ & 0.35391 & 5.2888 & \\
\hline C & 1.281352 & 2.551087 & 3.30 \\
\hline $\mathrm{H}$ & 1.391539 & 2.250537 & 4.3 \\
\hline $\mathrm{C}$ & 1.157583 & 1.55 & 2.3 \\
\hline C & 0.997078 & 1.922553 & 0.97886 \\
\hline C & 1.133038 & 0.095242 & 2.73 \\
\hline $\mathrm{H}$ & 0.241663 & -0.389278 & 2.3 \\
\hline $\mathrm{H}$ & 1.070787 & 0.050734 & 3.8319 \\
\hline C & 3.589595 & -0.172617 & 2.65 \\
\hline $\mathrm{H}$ & 3.457191 & 0.9043 & 2.8 \\
\hline $\mathrm{H}$ & 3.944472 & -0.580129 & 3.611 \\
\hline C & 4.678341 & -0.337038 & 1.61 \\
\hline $\mathrm{C}$ & 6.029728 & -0.315837 & 1.9 \\
\hline $\mathrm{H}$ & 6.312601 & -0.240681 & 3.00975 \\
\hline $\mathrm{C}$ & 6.994919 & -0.394523 & 0.9636 \\
\hline $\mathrm{H}$ & 8.050376 & -0.383124 & 1.2 \\
\hline $\mathrm{C}$ & 6.588055 & -0.491093 & -0.36743 \\
\hline $\mathrm{H}$ & 7.306334 & -0.555120 & -1.17707 \\
\hline $\mathrm{C}$ & 5.225448 & -0.507973 & -0.645 \\
\hline $\mathrm{H}$ & 4.833016 & -0.571723 & -1.65471 \\
\hline O & 2.592047 & -0.374537 & -2.104 \\
\hline $\mathrm{H}$ & 2.011156 & 0.221241 & -2.62 \\
\hline $\mathrm{C}$ & -4.934088 & -1.693848 & 0.96382 \\
\hline $\mathrm{C}$ & -5.141903 & -1.334550 & 2.29606 \\
\hline $\mathrm{C}$ & -4.065333 & -1.166540 & 3.17 \\
\hline C & -2.776539 & -1.348585 & 2.69790 \\
\hline C & -2.537656 & -1.698413 & 1.35065 \\
\hline $\mathrm{C}$ & -3.641781 & -1.876659 & 0.48961 \\
\hline $\mathrm{H}$ & -5.792050 & -1.830827 & 0.31692 \\
\hline $\mathrm{H}$ & -4.257262 & -0.895678 & 4.20206 \\
\hline $\mathrm{H}$ & -1.924187 & -1.226985 & 3.35765 \\
\hline $\mathrm{H}$ & -3.482230 & -2.169378 & -0.53841 \\
\hline $\mathrm{N}$ & -6.500002 & -1.129065 & 2.77966 \\
\hline O & -6.652395 & -0.831124 & 3.96779 \\
\hline 0 & -7.427201 & -1.258043 & 1.97409 \\
\hline
\end{tabular}

